\title{
EPOS LHC: Test of collective hadronization with data measured at the CERN Large Hadron Collider
}

\author{
T. Pierog* \\ Karlsruhe Institute of Technology (KIT) - Campus North - Institut für Kernphysik (IKP), Karlsruhe, Germany \\ Iu. Karpenko \\ Bogolyubov Institute for theoretical Physics, Kiev, Ukraine \\ and Frankfurt Institute for Advanced Studies, Frankfurt am Main, Germany
}

J. M. Katzy and E. Yatsenko

DESY, Hamburg, Germany

K. Werner

SUBATECH, University of Nantes - IN2P3/CNRS - EMN, Nantes, France

(Received 9 April 2015; published 14 September 2015)

\begin{abstract}
EPOS is a Monte Carlo event generator for minimum bias hadronic interactions, used for both heavy ion interactions and cosmic ray air shower simulations. Since the last public release in 2009, the Large Hadron Collider (LHC) experiments have provided a number of very interesting data sets comprising minimum bias $p-p$, $p-\mathrm{Pb}$, and $\mathrm{Pb}-\mathrm{Pb}$ interactions. We describe the changes required to the model to reproduce in detail the new data available from the LHC and the consequences in the interpretation of these data. In particular we discuss the effect of the collective hadronization in $p$ - $p$ scattering. A different parametrization of flow has been introduced in the case of a small volume with high density of thermalized matter (core) reached in $p$ - $p$ compared to large volume produced in heavy ion collisions. Both parametrizations depend only on the geometry and the amount of secondary particles entering in the core and not on the beam mass or energy. The transition between the two flow regimes can be tested with $p$ - $\mathrm{Pb}$ data. EPOS LHC is able to reproduce all minimum bias results for all particles with transverse momentum from $p_{\mathrm{t}}=0$ to a few $\mathrm{GeV} / c$.
\end{abstract}

DOI: 10.1103/PhysRevC.92.034906

\section{INTRODUCTION}

For many years the development of hadronic models able to reproduce with precision the particle production observed in minimum bias hadronic interactions has been a real challenge. These kinds of Monte Carlo (MC) models are not only important to test our knowledge of the physical processes involved, but they are also useful in other areas such as analysis of the detector acceptance in high energy physics (HEP) experiments or propagation of hadrons in the Universe or in the Earth's atmosphere for astrophysical applications. With the start of the Large Hadron Collider (LHC), a very large data set has become available. Although the models used for cosmic ray applications were able to predict the general behavior of these data [1], none of them was able to predict all minimum bias data consistently, and the models dedicated to HEP such as PYTHIA [2] failed to reproduce accurately distributions involving particles with very low transverse momenta and strange particle production.

In the MC generators commonly used in HEP, the soft part of the particle production, which dominates the minimum bias results, is dominantly calculated from perturbative QCD and to a small part via diffractive processes. The partons from the scattering process are showered and hadronized following the Lund string or the cluster fragmentation model. It was shown

*Tanguy.Pierog@kit.edu
PACS number(s): 25.75.-q, 24.10.Jv, 13.85.Hd

in [3] that at LHC energies hadronization cannot be done with a simple two-string model without multiple-scattering as for Intersecting Storage Rings (ISR) data. Many strings can be superimposed, and the hadronization cannot be treated like in an empty $e^{+} e^{-}$environment. The need of corrections to the hadronization models has also been acknowledged in $[4,5]$ where it is implemented in the form of so-called color reconnection.

In Fig. 1(a) we represent the classical description of $p-p$ scattering in HEP models. For $p$ - $p$ scattering at the LHC, "Projectile" and "Target" refer only to two opposite directions along the beam axis, but the system is naturally completely symmetric. The aim is to demonstrate that a model based on the complete chain of possible hadronic phase as represented in Fig. 1(b) commonly used for heavy ion (HI) collisions can lead to an improved description of minimum bias $p$ - $p$ data at the LHC. Such a model exists and has been actively developed for about 20 years. EPOS [6] is based on the parton-based Gribov-Regge theory [7] developed for NEXUS, which was based on the VENUS model [8] for soft interactions and the QGSJET model [9] for the semihard scattering.

Collective hydrodynamic flow seems to be well established in $\mathrm{HI}$ collisions at energies between 200 and $2760 \mathrm{~A} \mathrm{GeV}$, whereas $p$ - $p$ and $p-A$ collisions are often considered to be simple reference systems, showing normal behavior, such that deviations of HI results with respect to $p$ - $p$ or $p-A$ reveal new physics. Surprisingly, the first results from $p-\mathrm{Pb}$ at $5 \mathrm{TeV}$ on the transverse momentum dependence of azimuthal anisotropies 
(a)
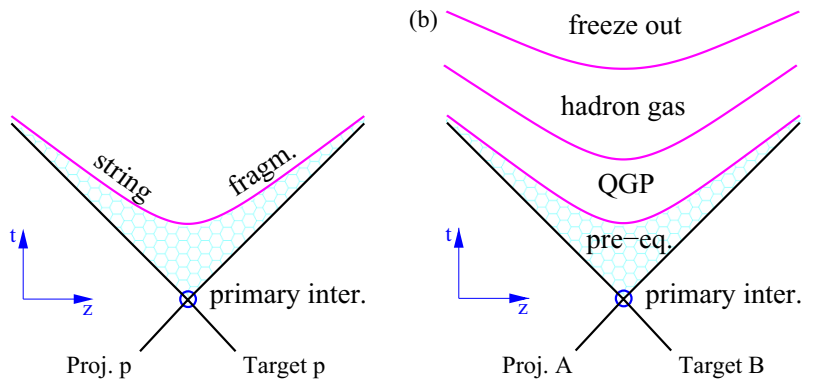

FIG. 1. (Color online) Space-time evolution of the particle production in a hadronic interaction. A hyperbola (line) represents particles with the same proper time. Panel (a) is the standard approach for $p$ - $p$ scattering while (b) is a more complete treatment used usually for HI collision.

and particle yields are very similar to the observations in $\mathrm{HI}$ scattering [10-13], and hence the question of "collective behavior in a small system" is heavily discussed. This discussion is mainly based on azimuthal anisotropies; in this sense our work is complementary since we are focusing on the consequences of radial flow in the lighter hadronic system: $p$ - $p$.

In this paper we will show how EPOS 1.99 [14] released in 2009 has been changed to reproduce in detail LHC data from various experiments. In Sec. II the basic principle of the model is discussed before a comparison to data in Sec. III with the updated version EPOS LHC (v3400). In particular the role of the collective flow which is changed compared to the one used in EPOS 1.99 is demonstrated. Tests with $p$ - $\mathrm{Pb}$ and $\mathrm{Pb}-\mathrm{Pb}^{\prime}$ data are shown in Sec. IV, and finally the difference to the PYTHIA model is discussed in Sec.V. Only LHC data are discussed here but EPOS LHC is tuned (with a single parameter set) to reproduce any kind of hadronic interactions from $h-A$ to $A-B$ where $h$ can be $\pi, K$, or $p$ and $A$ or $B$ range from 1 to 210 nucleons. The energy range is from $40 \mathrm{GeV}$ in the laboratory frame to more than $1000 \mathrm{TeV}$ center-of-mass (cms) energy (about $10^{21} \mathrm{eV}$ in the laboratory frame).

The EPOS version EPOS LHC v3400 presented here differs from EPOS 2.x [15] and EPOS 3.x [16] (under development) in that it does not take advantage of the complete 3D hydro calculation followed by the hadronic cascade done in EPOS 2 or 3 , but it is a released version which is freely available for any user [17]. The fast covariant approach used in EPOS 1.99 is still used but with an improved flow parametrization as described later. The main reason to have different versions is that for a $\mathrm{Pb}-\mathrm{Pb}$ central event EPOS 2 or 3 needs about one hour while EPOS LHC will generate it in a few tens of seconds, and EPOS LHC is not under development any more (public stable version). As a consequence EPOS LHC has more parameters (and less predictive power) than EPOS 2 or 3 [18-20] and should not be used for a precise study of $p_{\mathrm{t}}$ distributions or particle correlations in $\mathrm{HI}$ collisions, but is a good alternative model for $p-p$ and $p-A$ minimum bias analysis.

\section{UPDATE OF THE EPOS 1.99 MODEL}

\section{A. Basic principles of EPOS 1.99}

Nucleus-nucleus scattering - even proton-proton - amounts to many elementary collisions happening in parallel. Such

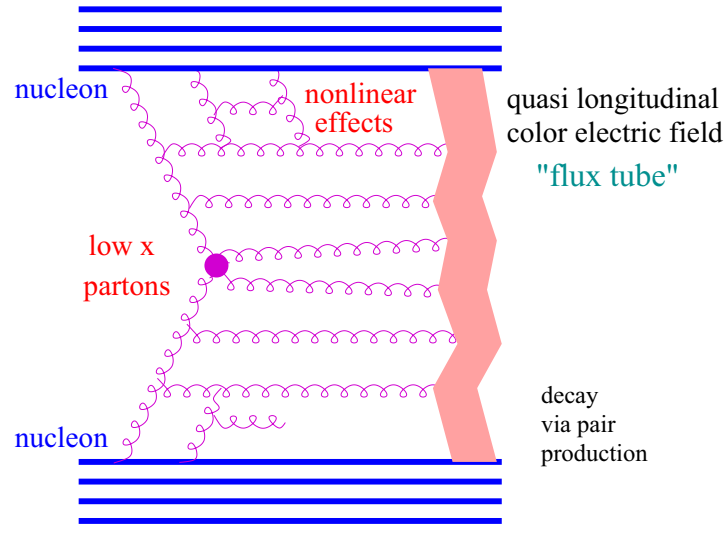

FIG. 2. (Color online) Elementary interaction in the EPOS model.

an elementary scattering is the so-called "parton ladder," see Fig. 2, also referred to as cut Pomeron [6].

A parton ladder represents parton evolutions from the projectile and the target side towards the center $(\operatorname{small} x$ ). The evolution is governed by an evolution equation, in the simplest case according to Dokshitzer-Gribov-Lipatov-Altarelli-Parisi (DGLAP). In the following we will refer to these partons as "ladder partons," to be distinguished from "spectator partons" to be discussed later. It was realized a long time ago that such a parton ladder may be considered as a quasilongitudinal color field, a so-called "flux tube" [15], conveniently treated as a relativistic string. The intermediate gluons are treated as kink singularities in the language of relativistic strings, providing a transversely moving portion of the object. This flux tube decays via the production of quark-antiquark pairs, creating in this way fragments, which are identified with hadrons.

The technical details of the consistent quantum mechanical treatment of the multiple scattering with energy sharing between the parallel scatterings can be found in [7]. Hard scale independent correction factors are added to the bare amplitude of the Pomeron to control the rise of the cross section at high energy and the multiplicity in HI collisions. The treatment of these nonlinear effects at high energy is explained in [6]. We do not discuss this part of the model here since very little change has been made compared to EPOS 1.99. Another article will cover the update of EPOS for the initial part of the collision (diffraction, string ends, and remnants).

\section{B. Collective hadronization in EPOS 1.99}

Statistical hadronization models have been used for many years to describe particle production in heavy ion collisions, but also to some extent in small systems such as $p$ - $p$ collisions [21-23]. Our approach is different in two ways: Whereas conventional hadronization models are done in the canonical framework, we are using a microcanonical scheme, with full conservation of everything (energy-momentum, flavor). The other difference concerns the object to decay: In our case, we use a dynamical model (the basic EPOS model) to construct a droplet of matter that is not uniform (for example the baryonic density may vary along the longitudinal direction), whereas conventional hadronization models assume some (uniform) object. Also these models only predict yields, whereas we 
produce distributions. In this sense our model is similar to socalled blast-wave models [24], where statistical hadronization is based on a parametrized freeze-out surface (but here again the canonical framework is used).

First of all, it is important to note that the initial conditions for hadronization in EPOS are based on strings, not on partons. Here "initial condition" refers to the state of the system after the initial and final state radiation of the jets when partons hadronize in HEP models and before possible parton or hadron rescattering like in $\mathrm{HI}$ collisions (final state interactions). As explained in the previous section, the initial scatterings lead to the formation of strings, which break into segments, which are usually identified with hadrons. Then one considers the situation at an early proper time $\tau_{0}$, long before the hadrons are formed: one distinguishes between string segments in dense areas (more than some critical density $\rho_{0}$ segments per unit volume) and those in low density areas. The high density areas are referred to as core, the low density areas as corona [25]. The corona is important for certain aspects such as the centrality dependence of all observables in HI collisions. Here it will correspond to unmodified string fragmentation as in usual HEP models and will dominate at large rapidity and in low multiplicity events. In this section we will focus on the core part which is unique in EPOS and provides interesting effects not taken into account in other HEP models (which are all "corona"-like).

Based on the four-momenta of the string segments which constitute the core, a matrix in $(x, y, \eta)$ of the segment density is formed. The core is made of different clusters in each $\eta$ bin to keep the local energy density distribution, and each cluster is hadronized via a microcanonical procedure with an additional longitudinal and radial flow exactly as described in [25]. The whole procedure perfectly conserves energy, momentum, and flavors. The free parameters used in this process such as strangeness or baryon production correction factor and energy density at freeze-out can be fixed using HI data on particle production. The mass $M$ of each cluster is defined as

$$
M=\sqrt{\left(\sum_{i} E_{i}\right)^{2}-\left(\sum_{i} \vec{P}_{i}\right)^{2}}
$$

where $i$ is the index of all segments forming the cluster and $\left(E_{i}, \vec{P}_{i}\right)$ the four-momentum vector of a segment.

In each event a part of the string segments hadronizes normally (corona), and a part is used to create a core with a collective hadronization as represented in Fig. 3. The core appears only if the local density of string segments is high enough. This limit is of course easily reached in the case of central HI collisions at the Relativistic Heavy Ion Collider (RHIC) or the LHC [or even the Super Proton Synchrotron (SPS)] because of the large number of pairs of nucleons suffering an inelastic interaction.

But in fact the multiple scattering of partons for a given pair of nucleons can be enough to create many strings which will overlap since the distance between partons is very small. At $7 \mathrm{TeV}$ in $p$ - $p$ it is easily possible to produce more than five flux tubes leading to the production of much more than ten strings very close to each other in the transverse plane and overlapping around $\eta=0$. Since a string produces more

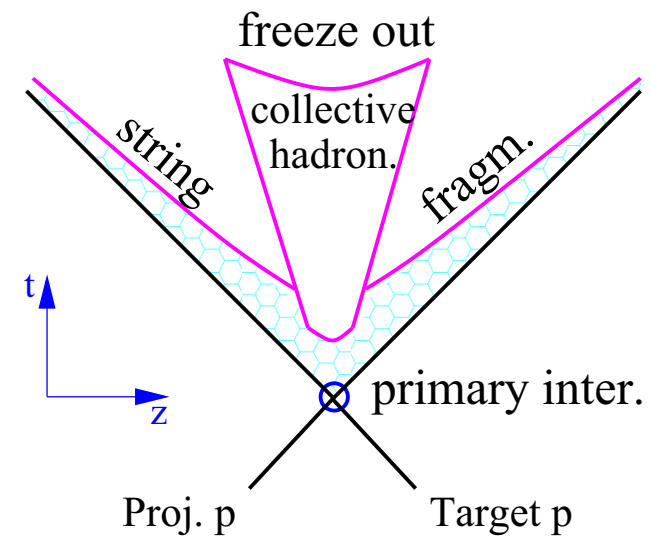

FIG. 3. (Color online) Schematic view of the space-time evolution of the particle production in a hadronic interaction in EPOS 1.99 or EPOS LHC. A hyperbola (line) represents particles with the same proper time. The same treatment is used for $p-p$ or $A-B$ but the collective hadronization, which can be local, is simplified compared to the full HI picture (done in EPOS 2 or 3).

than a minimum given number of particles, a large number of strings implies a large multiplicity. So, plotting the fraction of final particles produced by core decay as a function of the multiplicity of charged particles with $|\eta|<2.4$ as shown in Fig. 4, we can notice that even for the average multiplicity at $7 \mathrm{TeV}$ (solid line), which is about 30 (with four to six strings on average), about $30 \%$ of the particles come from the core. The rest are produced directly by the string fragmentation in the corona region where string segments do not overlap. At $900 \mathrm{GeV}$ (dashed line), at the average multiplicity (about 15), this fraction is close to 0 . But for the same number of particles, about the same ratio is reached at both energies.

Of course not all particles are completely absorbed in the dense area. We define a parameter $p_{\mathrm{t}}^{\text {cut }}$ above which a particle will simply lose part of its momentum in the core but will survive as an independent particle produced by a string (typically high $p_{\mathrm{t}}$ particles from jets). Soft particles with $p_{\mathrm{t}}<p_{\mathrm{t}}^{\text {cut }}$

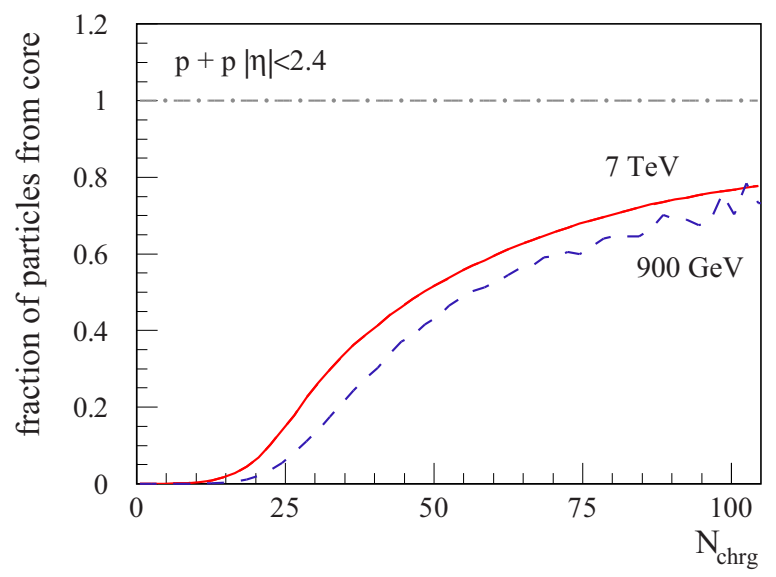

FIG. 4. (Color online) Fraction of charged particles with $|\eta|<$ 2.4 coming from the core as a function of the total number of charged particles with $|\eta|<2.4$. The solid line is for simulation with EPOS at $7 \mathrm{TeV}$ and the dashed line for $900 \mathrm{GeV} p$ - $p$ scattering. 
are completely absorbed and form the core. The energy loss depends on the system size and follows formulas from [26,27].

\section{Collective flow}

\section{Flow definitions in EPOS 1.99}

Since string segments show a Bjorken-fluid like behavior, and clusters are formed from these segments, clusters are considered to be collectively expanding:

(1) Some transverse expansion is driven by the maximal radial rapidity $y_{\mathrm{rad}}$ if the total mass of the core $M_{\text {core }}=$ $\sum_{\text {clusters }} M$ is larger than $M_{\min }=3 \mathrm{GeV} / c^{2}$.

(2) Bjorken-like expansion in the longitudinal direction is driven by the maximal longitudinal rapidity $y_{\text {long }}$ for each individual cluster whose mass $M$ is larger than the minimum mass $M_{\min }$.

In [25], it is assumed that the clusters hadronize at some given energy density $\varepsilon_{\text {hadr }}$, having acquired at that moment a collective radial flow, with a linear radial rapidity profile from inside to outside, characterized by $y_{\mathrm{rad}}$. In addition, an azimuthal asymmetry was imposed by multiplying the $x$ and $y$ components of the flow four-vector-velocity with $1+\min \left(\epsilon, f_{\text {ecc }}\right)$ and $1-\min \left(\epsilon, f_{\text {ecc }}\right)$, where $\epsilon$ is the initial spacial eccentricity, $\epsilon=\left\langle\left(y^{2}-x^{2}\right) /\left(y^{2}+x^{2}\right)\right\rangle$, and $f_{\text {ecc }}=$ 0.5 a parameter. By imposing radial flow, the cluster mass had to be rescaled as

$$
M \rightarrow M \times 0.5 y_{\mathrm{rad}}^{2} /\left(y_{\mathrm{rad}} \sinh y_{\mathrm{rad}}-\cosh y_{\mathrm{rad}}+1\right),
$$

in order to conserve energy, and for the longitudinal flow we have in addition

$$
M \rightarrow M \times y_{\text {long }} / \sinh y_{\text {long }} .
$$

\section{New features in EPOS LHC}

As a consequence of the rescaling due to collective flows, and in particular the radial flow, the number of secondary particles produced by the clusters is reduced. In the case of consistent treatment of cross section and particle production as in EPOS, this property is needed in the case of HI collisions where fewer particles are observed than produced by the model without final state interactions. Indeed, a proper hydro treatment as in EPOS 2 or 3 requires a large multiplicity in the initial state to finish with the correct multiplicity after a long evolution of the large volume of the core. We will call it the nuclear AA flow, characterized by the maximal radial rapidity $y_{\text {rad }}^{\text {AA }}$.

But in the case of a light system, such as $p$-p, using EPOS 2 or 3 with a realistic treatment of the hydrodynamical evolution with proper hadronization, such an effect was not observed [16,20,28]. In that case the large flow comes from the quick expansion of the very small volume of the core. As a consequence, in EPOS LHC we introduced a different type of radial flow in the case of a very dense system in a small volume (where the critical energy density is reached because of multiple scattering between partons in a single pair of nucleons as in $p-p$ ). For this "pp flow," characterized by the maximal radial rapidity $y_{\mathrm{rad}}^{\mathrm{pp}}$, the mass of the cluster $M$ is not changed before hadronization (multiplicity is conserved) but the energy conservation is imposed by a simple rescaling of the total momentum $P$ (larger $p_{\mathrm{t}}$ are compensated by smaller $p_{\mathrm{z}}$ ) after the radial boost. Of course a smooth transition is needed between the two kinds of systems and the transition is observed in $p$ - $A$ interactions.

In EPOS 1.99, $y_{\text {rad }}$ was parametrized as function of the system energy and size as $y_{\mathrm{rad}}=y_{\mathrm{rad}}^{\mathrm{mx}}+y_{\mathrm{rad}}^{\mathrm{mi}} \ln \left(1 .+\sqrt{s / N_{\text {pair }}}\right)$, where $N_{\text {pair }}$ is the number of possibly interacting pairs of nucleons and $y_{\text {rad }}^{\mathrm{mx}}$ and $y_{\text {rad }}^{\mathrm{mi}}$ are parameters. While the evolution with $N_{\text {pair }}$ was safe and easy to test with HI data at the SPS and the RHIC, the evolution with energy especially for $N_{\text {pair }}=1$ $(p-p)$ could lead to wrong extrapolation at high energy.

In the paper on identified particle spectra from the CMS Collaboration [29], data show that the increase of the $\left\langle p_{\mathrm{t}}\right\rangle$ as a function of the multiplicity does not depend on the center-of-mass energy but increases with multiplicity. Because this effect is directly linked to the radial flow intensity in our approach [28], it is natural to parametrize all flows as functions of the total mass $M_{\text {core }}$ which is directly linked to the final multiplicity. We get for the longitudinal flow

$$
y_{\text {long }}=y_{\text {long }}^{\mathrm{mx}} \ln \left[\exp \left(\frac{y_{\text {long }}^{\mathrm{mi}}}{y_{\text {long }}^{\mathrm{mx}}}\right)+\frac{M_{\text {core }}}{M_{\text {min }}}\right] \text {, }
$$

the AA radial flow

$$
y_{\mathrm{rad}}^{\mathrm{AA}}=y_{\mathrm{rad}}^{\mathrm{mx}} \ln \left(\frac{M_{\text {core }}}{M_{\mathrm{min}}}\right),
$$

and the pp radial flow

$$
y_{\text {rad }}^{\mathrm{pp}}=y_{\text {rad }}^{\mathrm{px}} \ln \left(\frac{M_{\text {core }}}{M_{\text {min }}}\right),
$$

where $y_{\text {rad }}^{\mathrm{mx}}$ is the parameter fixing the radial flow and the multiplicity in HI, and $y_{\text {rad }}^{\mathrm{px}}$ determines the evolution of $\left\langle p_{\mathrm{t}}\right\rangle$ as a function of the multiplicity in $p$ - $p$. The longitudinal flow being weaker, $y_{\text {long }}^{\mathrm{mx}}$ and $y_{\text {long }}^{\mathrm{mi}}$ are parameters which are used to do a fine tuning of the multiplicity in both $p-p\left(y_{\text {long }}^{\mathrm{mi}}\right)$ and HI interactions ( $y_{\text {long }}^{\mathrm{mx}}$ ). $M_{0}=3 \mathrm{GeV} / c^{2}$ is the minimum mass to have flow.

These definitions are good if we have pure AA flow (slow expansion of a large volume) or pure pp flow (fast expansion of a small volume). For peripheral nuclear collision or $p-A$ interactions we have to decide whether $y_{\text {rad }}^{\mathrm{pp}}$ (high density of string segments coming from a single nucleon pair as in $p-p$ ) or $y_{\text {rad }}^{\text {AA }}$ (high density of string segments coming from many different nucleon pairs as in central HI) should be used. So for pp flow parametrization one can define

$$
M_{\mathrm{pp}}=\min \left(1, f_{\mathrm{pp}} \frac{N_{\mathrm{max}}^{p p}}{N_{\mathrm{tot}}}\right) M_{\text {core }},
$$

where $N_{\max }^{p p}$ is the maximum number of segments used in the core coming from a unique nucleon-nucleon pair and $N_{\text {tot }}$ is the total number of segments forming the core. So $N_{\max }^{p p} / N_{\text {tot }}$ is simply the fraction of segments coming from the pair contributing the most to the core. $f_{\mathrm{pp}}=1.3$ is a parameter which can be tuned in order to have the same $\left\langle p_{\mathrm{t}}\right\rangle$ as a function of multiplicity in $p-p$ or $p$ - $A$ at low multiplicity (no data needed). In other words $M_{\mathrm{pp}}$ is simply the mass of the part of the core coming from the pair of nucleons with the largest 
multiple scattering. As a consequence Eq. (6) can be rewritten as

$$
y_{\text {rad }}^{\mathrm{pp}}=y_{\mathrm{rad}}^{\mathrm{px}} F_{\mathrm{pp}} \ln \left(\frac{M_{\mathrm{pp}}}{M_{\mathrm{min}}}\right),
$$

where $F_{\mathrm{pp}}=\min \left(1,2\left\langle N^{p p}\right\rangle / N_{\max }^{p p}\right)^{2}$, with $\left\langle N^{p p}\right\rangle$ being the average number of segments going to the core per participating pair of nucleons, is a normalization factor going to 0 in the case of HI collisions. As expected $F_{\mathrm{pp}}=1$ and $M_{\mathrm{pp}}=M_{\text {core }}$ in the case of $p$ - $p$ scattering. Using Eq. (5), we have two independent definitions for $y_{\mathrm{rad}}^{\mathrm{pp}}$ and $y_{\mathrm{rad}}^{\mathrm{AA}}$. Since $y_{\mathrm{rad}}^{\mathrm{pp}}>y_{\mathrm{rad}}^{\mathrm{AA}}$ for a small system (small number of nucleon pairs) and $y_{\text {rad }}^{\text {pp }}<y_{\text {rad }}^{\mathrm{AA}}$ for a large system (when $\left\langle N^{p p}\right\rangle \ll N_{\text {max }}^{p p} ; F_{\mathrm{pp}} \rightarrow 0$ ), we simply use the pp flow when $y_{\text {rad }}^{\mathrm{pp}}>y_{\text {rad }}^{\mathrm{AA}}$ and the AA flow otherwise.

Since the flows depend only on $M_{\text {core }}$ all parameters can be fixed using LHC data only ( $p-p$ and $\mathrm{Pb}-\mathrm{Pb}$ ) but the results are checked with RHIC and SPS data.

\section{COMPARISON WITH LHC DATA}

In this section we will see how LHC data can be described by EPOS LHC.

\section{A. Cross section}

The most fundamental parameters of the EPOS model are fixed by comparing the cross sections calculated from the single scattering amplitude with the measured data. Thanks to the TOTEM experiment [30], total, inelastic, and elastic cross sections are known now with a high precision and can be used to constrain the model at high energy. As shown in Fig. 5, after retuning of the parameters fixing the nonlinear effects in EPOS LHC, the total, inelastic, and elastic cross sections can be well reproduced (solid line).

Compared to EPOS 1.99 (dashed line), the result corresponds to an increase of the inelastic cross section due to a larger amplitude of the parton ladder. As a consequence the multiplicity predicted by the model should increase at the LHC.

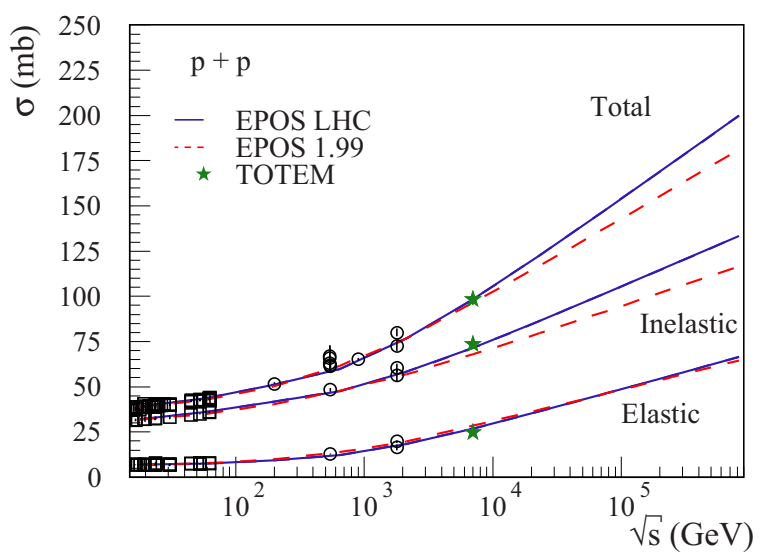

FIG. 5. (Color online) Total, inelastic, and elastic $p$ - $p$ cross sections calculated with EPOS LHC (solid line) and EPOS 1.99 (dashed line). Points are data from [31] and the stars are the LHC measurements by the TOTEM experiment [30].

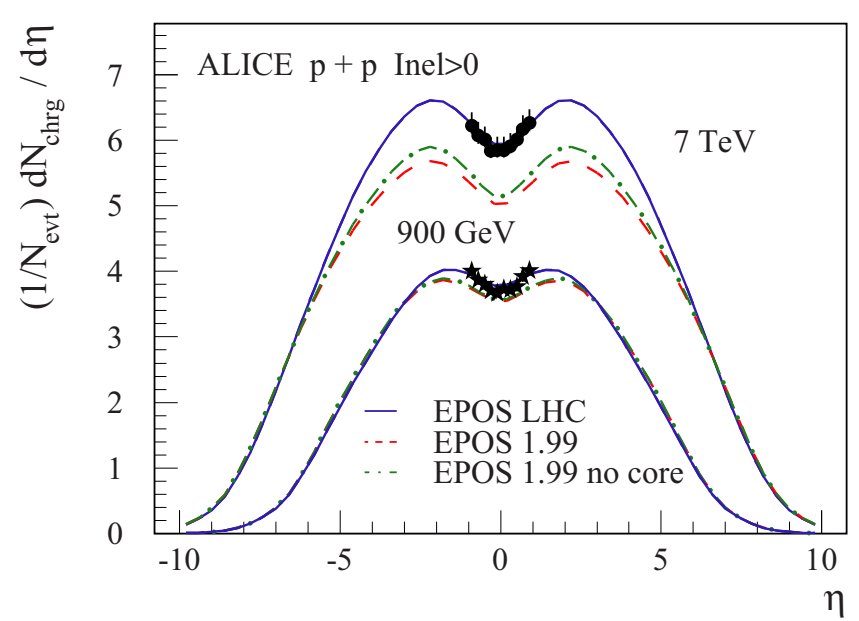

FIG. 6. (Color online) Pseudorapidity distribution of charged particles for events with at least one charged particle with $|\eta|<1$ for $p$ - $p$ interactions at $900 \mathrm{GeV}$ and $7 \mathrm{TeV}$. Simulations with EPOS LHC (solid line), EPOS 1.99 (dashed line), and EPOS 1.99 without core production (dash-dotted line) are compared to data points from the ALICE experiment [32]

\section{B. Particle production}

As we can see in Fig. 6, showing the comparison of EPOS with the pseudorapidity distribution of charged particles from ALICE data [32], the mean multiplicity is indeed larger in EPOS LHC (solid line) compared to EPOS 1.99 (dashed line). From the simulations with EPOS 1.99 without core formation (dashdotted line), we can check that this is not due to the corrected flow. The increase in multiplicity is a direct consequence of the consistent treatment [7] of the cross section and the particle production in the EPOS framework. By adjusting the parameters to get the correct cross section, we obtain naturally the correct multiplicity.

Looking at the corresponding multiplicity distribution at $7 \mathrm{TeV}$ from the ALICE experiment [33] in Fig. 7, we can observe the effect of the corrected flow on the tail of the

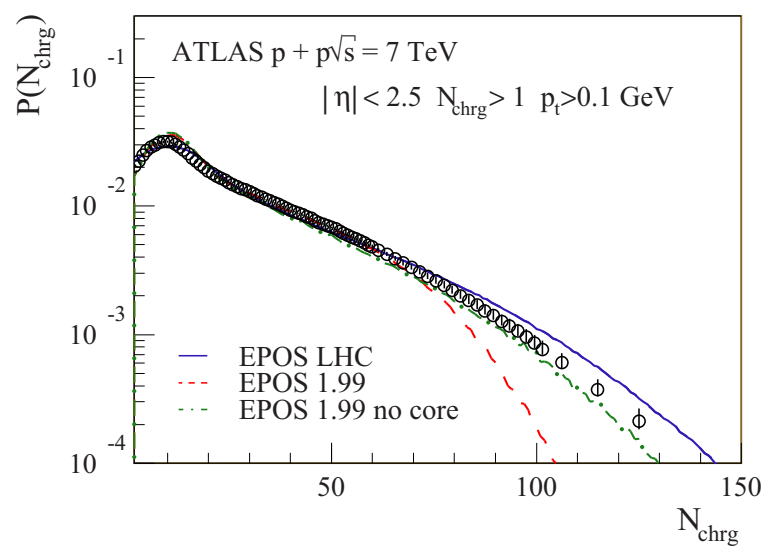

FIG. 7. (Color online) Multiplicity distribution of charged particles with $p_{\mathrm{t}}>100 \mathrm{MeV}$ and $|\eta|<2.5$ for $p$ - $p$ interactions at $7 \mathrm{TeV}$. Simulations with EPOS LHC (solid line), EPOS 1.99 (dashed line), and EPOS 1.99 without core production (dash-dotted line) are compared to data points from the ATLAS Collaboration [33]. 


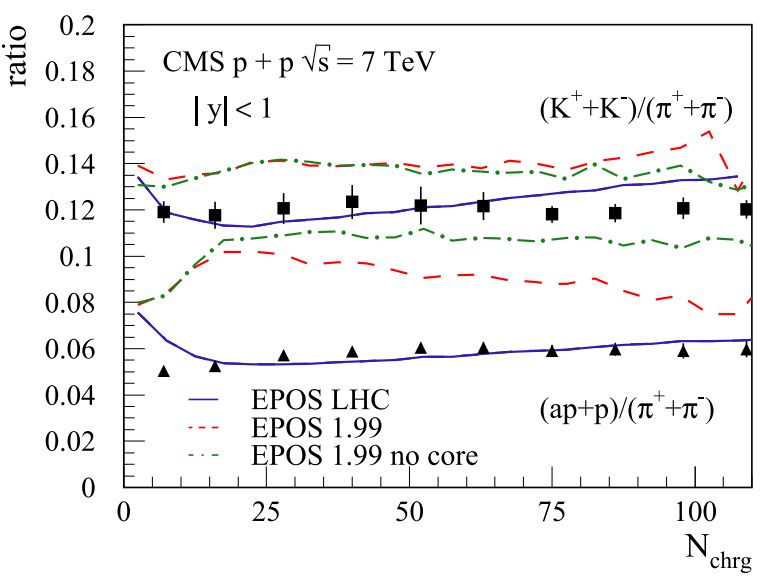

FIG. 8. (Color online) Ratio of particle yield as a function of charged particle multiplicity for $|y|<1$ for non-single-diffractive (NSD) $p$ - $p$ scattering at $7 \mathrm{TeV}$. Simulations are done with EPOS LHC (solid line), EPOS 1.99 (dashed line), and EPOS 1.99 without core production (dash-dotted line). Points are data from the CMS experiment [29].

distribution. In EPOS 1.99 (dashed line) for the events with a large multiplicity, the flow effect was strong and was reducing the total number of particles suppressing events with large multiplicities. Without core formation in EPOS 1.99 (dash-dotted line), the results were already reasonable, and now in EPOS LHC the tail is well reproduced even with core formation (solid line).

Thanks to CMS data on identified charged particle ratios at midrapidity [29] we could identify a problem in EPOS 1.99 concerning the production of baryon-antibaryon pairs (and strangeness) in string fragmentation (dashed line in Fig. 8) which were artificially increased at high energy (by a factor of 2 for diquark production). After correction, and using the same parameters as in $e^{+} e^{-}$string fragmentation, the data are now well reproduced for both kaon and (anti)proton production by EPOS LHC (solid line in Fig. 8). The effect of the core formation on these types of particles is small as we can see for the kaon ratio comparing EPOS 1.99 with and without core formation (dashed-dotted line in Fig. 8). For the proton ratio using EPOS 1.99 we can clearly see the transition from particle production dominated by the (wrong) string fragmentation at low $N_{\text {ch }}$ to particle production dominated by cluster decay at large $N_{\text {ch }}$ were the proton-to-pion ratio is correct.

With correct string fragmentation parameters as in EPOS LHC, the transition from pure strings to clusters is difficult to observe with these types of "light quark" particles. We will see in the next section that the effect of the final state interaction is much larger for multistrange baryons.

\section{Final state interaction}

We just reported in Sec. III B that, when everything is treated correctly, the effect of a possible statistical decay phase with a radial flow due to parton or hadron reinteraction after the initial state interaction is difficult to observe in (light) particle multiplicity with a long lifetime. We will see in this section that specific observables show clear indications that indeed

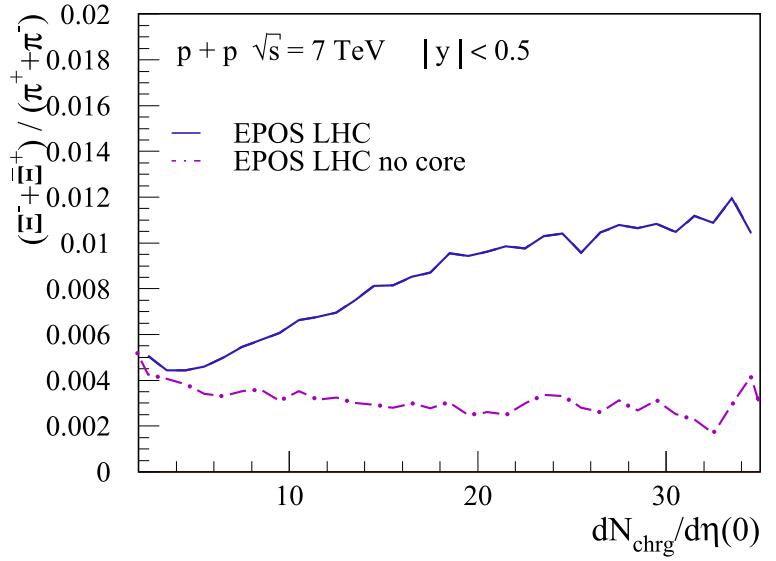

FIG. 9. (Color online) Ratio $\Xi / \pi$ as a function of particle density at midrapidity from EPOS LHC simulations with (solid line) and without core production (dash-dotted line).

final state interaction effects clearly help to reproduce $p$ - $p$ particle production.

\section{Particle ratio}

One of the effects of the reinteraction is that the particle production is not only coming from string fragmentation (where all the parameters are supposed to be fixed by data on $e^{+} e^{-}$ particle production) but also from a phase were particles are produced statistically. In such a hadronization process corresponding to a hadron gas in equilibrium, strangeness production is not suppressed. In practice the equilibrium is not necessarily reached, and some suppression (as a free parameter) can be introduced, but it is shown in [34] that the strangeness production is much larger (by about a factor of 2) in HI collisions (where the parameters for statistical hadronization are fixed) than in $e^{+}-e^{-}$interactions (where string fragmentation parameters are fixed). In [35] for instance, the hyperon-to-meson ratio is used as a possible proof of a mini quark-gluon plasma. In EPOS LHC, we can clearly see the transition from a pure string fragmentation to a statistically dominated hadronization, looking at the evolution of the multistrange baryon-to-pion ratio as a function of the multiplicity at midrapidity as shown Fig. 9. Without core formation (dash-dotted line), there is no strong evolution, while with core formation (solid line) the ratio increases almost linearly with the plateau height. The effect would be even larger with the $\Omega$ baryon.

Comparing, Fig. 10, $\Lambda^{0}+\bar{\Lambda}^{0}$ and $\Xi^{-}+\bar{\Xi}^{+}$rapidity distributions as measured by the CMS experiment [36] for NSD events with EPOS LHC simulation with (solid line) core formation, we observe a good agreement, while without (dash-dotted line) core formation the average $\Xi$ production is a factor of 2 lower.

\section{Transverse momentum}

The second main effect of the collective phase is the generation of a collective flow as described in Sec. II C. After cluster decay a random longitudinal Lorentz boost, whose maximal value is given by Eq. (4), and a radial Lorentz boost, whose maximal value is given by Eq. (8) and whose phase 


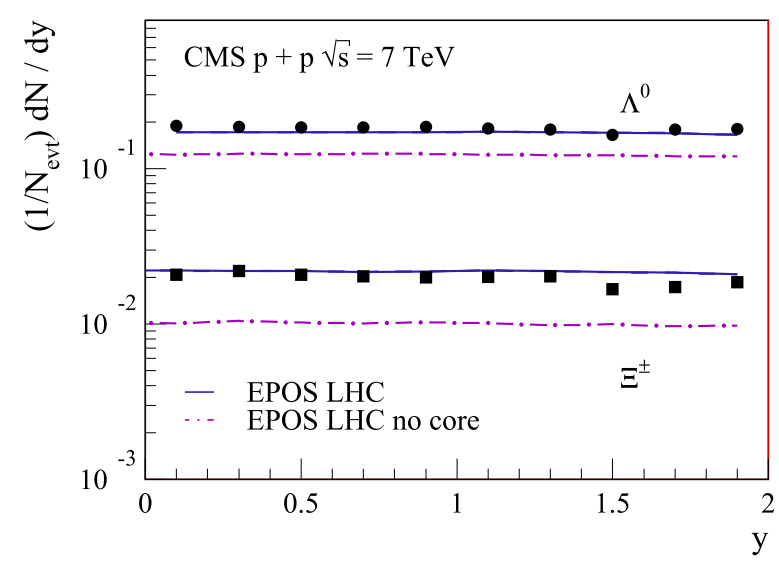

FIG. 10. (Color online) Strange baryon yield as a function of rapidity for non-single-diffractive (NSD) $p$ - $p$ scattering at $7 \mathrm{TeV}$. Simulations are done with EPOS LHC with core (solid line) and without core production (dash-dotted line). Points are data from the CMS experiment [36].

depends on cluster geometry, are applied to each particle. The effect is better observed in the evolution of the mean $p_{\mathrm{t}}$ as a function of the number of particles at midrapidity.

In Fig. 11, we first show that EPOS simulations without core formation (dash-dotted line) exhibit a flat behavior in the case of hard nondiffractive events $\left(N_{\mathrm{ch}}>25\right)$. It is easy to understand in term of string fragmentation even with multiple scattering since each string uses the same $\left\langle p_{\mathrm{t}}\right\rangle$. Then we can check that in the case of EPOS 1.99 (dashed line) the $\left\langle p_{\mathrm{t}}\right\rangle$ due to radial flow was extrapolated to a too large value at $7 \mathrm{TeV}$. Using Eq. (8) in EPOS LHC (solid line) and adjusting the parameter $y^{\mathrm{px}}$ to get the best fit at $900 \mathrm{GeV}$ and $7 \mathrm{TeV}$ of ATLAS data from [33], it is possible to get a very good description of the measurements. Since the radial boost is based on a Lorentz transformation, it depends on the total energy, thus on the mass, of each particle. The higher the mass is, the stronger the effect. It can be checked on the evolution of the mean $p_{\mathrm{t}}$ as a function

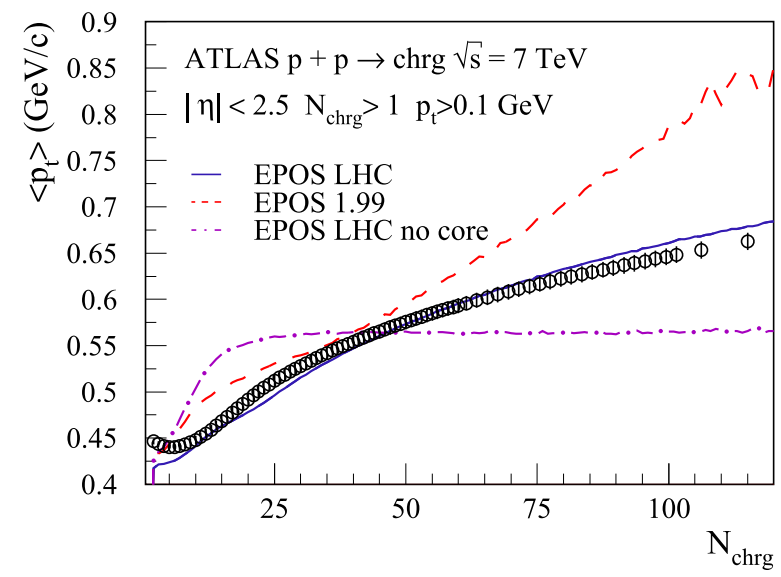

FIG. 11. (Color online) Average transverse momentum $\left\langle p_{\mathrm{t}}\right\rangle$ as a function of the number of charged particles for charged particles with $p_{\mathrm{t}}>0.1 \mathrm{GeV}$ and $|\eta|<2.5$ for $p$ - $p$ interactions at $7 \mathrm{TeV}$. Simulations are done with EPOS LHC (solid line), EPOS 1.99 (dashed line), and EPOS LHC without core production (dash-dotted line). Points are data from the ATLAS experiment [33].

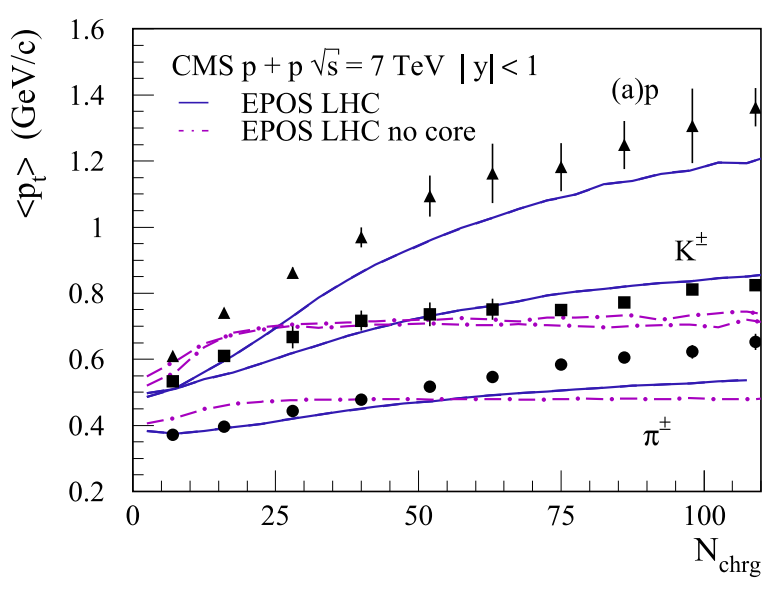

FIG. 12. (Color online) Average transverse momentum $\left\langle p_{\mathrm{t}}\right\rangle$ of identified particles $(\pi, K$, and $p$ ) as a function of the number of charged particles for particles with rapidity $|y|<1$ in $p$ - $p$ collisions at $7 \mathrm{TeV}$. Simulations are done with EPOS LHC with (solid line) and without (dash-dotted line) core formation. Points are data from the CMS experiment [29].

of the number of particles for identified particles $(\pi, K$, and $p$ ) as published by the CMS Collaboration in [29]. In Fig. 12 we can see that $\left\langle p_{\mathrm{t}}\right\rangle$ depends on the mass of the particles, and that EPOS LHC gives a reasonable description of the data when the flow is active (solid lines) while the standard string fragmentation gives a completely different behavior (dash-dotted line).

Comparing directly EPOS LHC simulations with the transverse momentum distribution measured by the ATLAS experiment [33] in minimum bias $p$ - $p$ interaction as in Fig. 13, we

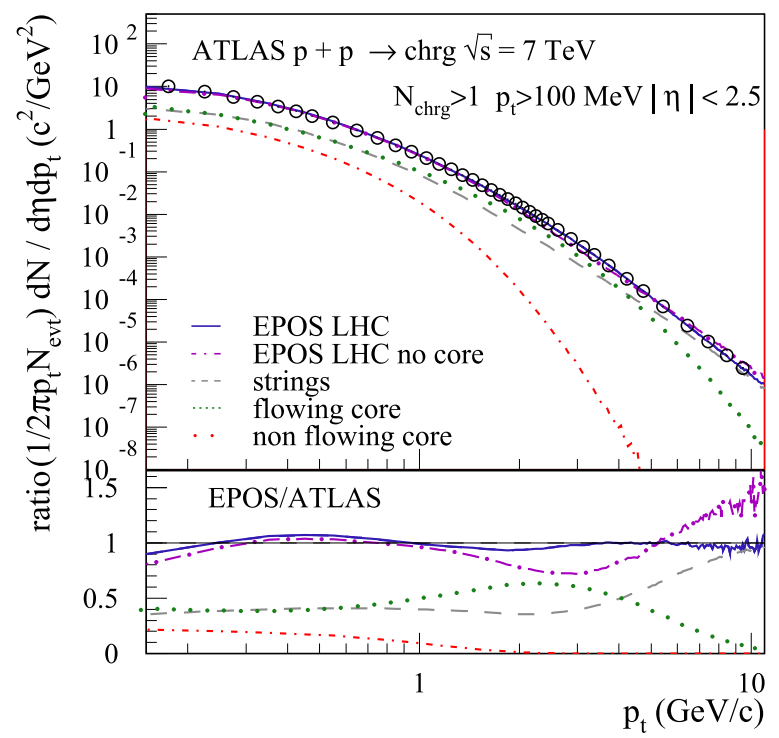

FIG. 13. (Color online) Transverse momentum $p_{\mathrm{t}}$ distribution of charged particles with $|\eta|<2.5$ produced in minimum bias $p$ - $p$ collisions at $7 \mathrm{TeV}$. Simulations are done with EPOS LHC with (solid line) and without (dash-dotted line) core formation. The contributions of particles coming from the core hadronization are shown as a dotted line while particles coming directly from string fragmentation are represented by a dashed line. Points are data from the ATLAS experiment [33]. 


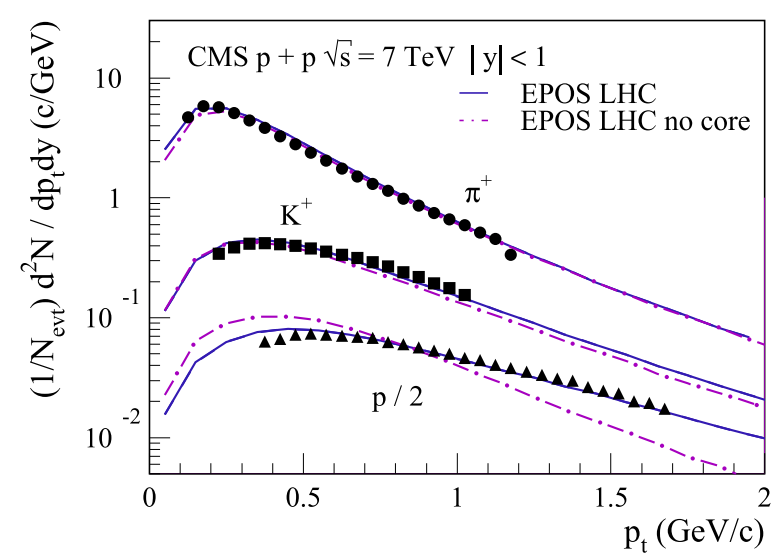

FIG. 14. (Color online) Transverse momentum distribution of identified particles $(\pi, K$, and $p)$ for $|y|<1$ for NSD $p$ - $p$ scattering at $7 \mathrm{TeV}$. Simulations are done with EPOS LHC with (solid line) and without (dash-dotted line) core formation. Points are data from the CMS experiment [29].

can see that the particles coming from the core hadronization with a radial flow (dotted line) will dominate the flux around $1-2 \mathrm{GeV} / c$, which is exactly the place where a deficit is observed when a model without flow (dash-dotted line) is compared to measured data. The position of the transition (and as a consequence of the shape of the $p_{\mathrm{t}}$ distribution) depends on the parameter $p_{\mathrm{t}}^{\text {cut }}$ whose best value is $1 \mathrm{GeV} / c$. At large $p_{\mathrm{t}}(>5 \mathrm{GeV} / c)$ the particles which are not completely absorbed into the high density region after string fragmentation dominate again.

In fact the effect is already clearly visible in the minimum bias transverse momentum distribution of identified particles. In Fig. 14 simulations both with (solid line) and without (dashdotted line) core formation can describe the $\pi p_{\mathrm{t}}$ spectrum from [29]. But when the mass increases, the deviation between the standard hadronization without flow and the data increases, while the simulations with collective hadronization give a good result. If we consider strange baryons which has even larger masses, it can be seen in Fig. 15 that the difference between

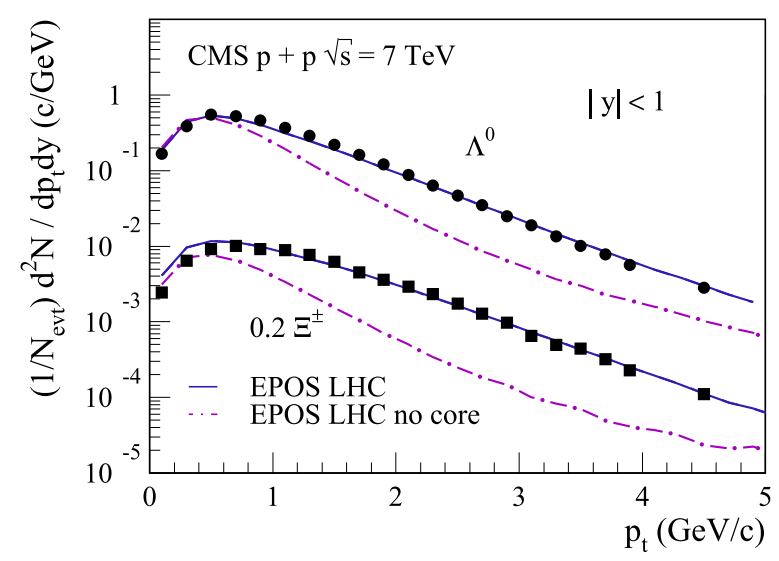

FIG. 15. (Color online) Transverse momentum distribution of strange baryons $(\Lambda$ and $\Xi)$ for $|y|<1$ for NSD $p$ - $p$ scattering at $7 \mathrm{TeV}$. Simulations are done with EPOS LHC with (solid line) and without (dash-dotted line) core formation. Points are data from the CMS experiment [36].

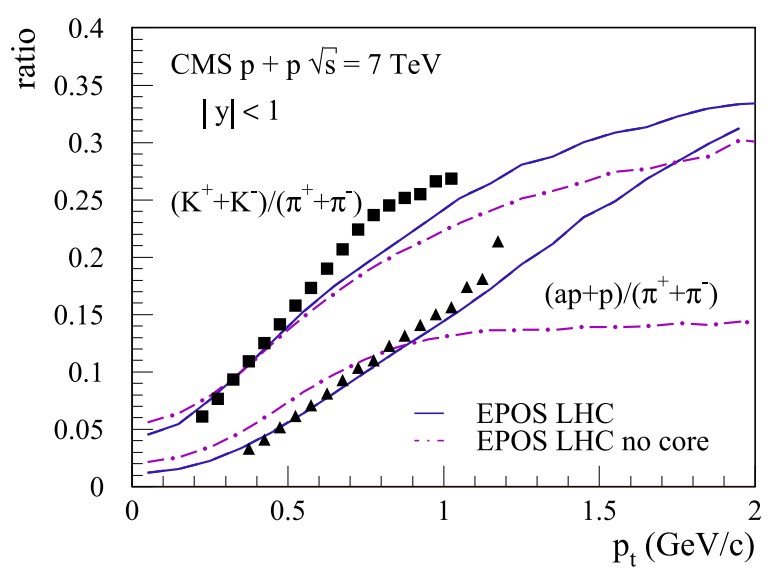

FIG. 16. (Color online) Ratio of particle yield as a function of transverse momentum for $|y|<1$ for NSD $p$ - $p$ scattering at $7 \mathrm{TeV}$. Simulations are done with EPOS LHC with (solid line) and without (dash-dotted line) core formation. Points are data from the CMS experiment [29].

the two approaches can be as large as a factor of 5 for cascade particles [36] where the flow effect combines with the yield effect described in the previous section.

Finally, using the ratios of kaons to pions and protons to pions as functions of the transverse momentum as plotted in Fig. 16, it can be clearly seen from data and simulations with (solid line) and without (dash-dotted line) core formation that the flow effect takes place only above $p_{\mathrm{t}}>1 \mathrm{GeV} / c$.

\section{HEAVY ION INTERACTIONS}

The EPOS model was originally designed for heavy ion collisions. Even if the retuned LHC version described in this paper is based on the simplified treatment of collective hadronization from [25] and not on the more sophisticated hydrodynamical treatment of [28], it is important to check the basic distributions for heavy systems.

\section{A. Lead-lead}

As explained in Sec. IIC, the so-called AA flow parametrization reduces cluster masses (and as a consequence the multiplicity of secondary particles) to increase the mean transverse momentum of the produced particles. As a consequence, both multiplicity and transverse momentum need to be taken into account when fixing the parameter $y_{\mathrm{rad}}^{\mathrm{mx}}$.

As can be seen in Figs. 17 and 18, it is possible to achieve a good description of both the pseudorapidity distribution of $\mathrm{Pb}-\mathrm{Pb}$ collisions at $2.76 \mathrm{TeV}$ with various centralities from the ATLAS and ALICE experiments [37,38] and the nuclear suppression factor of the most central events as measured by the ALICE experiment [39] for $p_{\mathrm{t}}<5 \mathrm{GeV} / c$. The large suppression observed in the simulations with EPOS LHC at larger $p_{\mathrm{t}}$ is due to a lack of hard scatterings during the initial stage of the nucleus-nucleus interaction. Indeed the screening effects used in EPOS [6] for a good description of soft processes in $p-p$ and $A-B$ scattering affect hard scales the same way as soft scales. In fact it has been shown now that such an initial stage suppression of hard processes is not observed in 


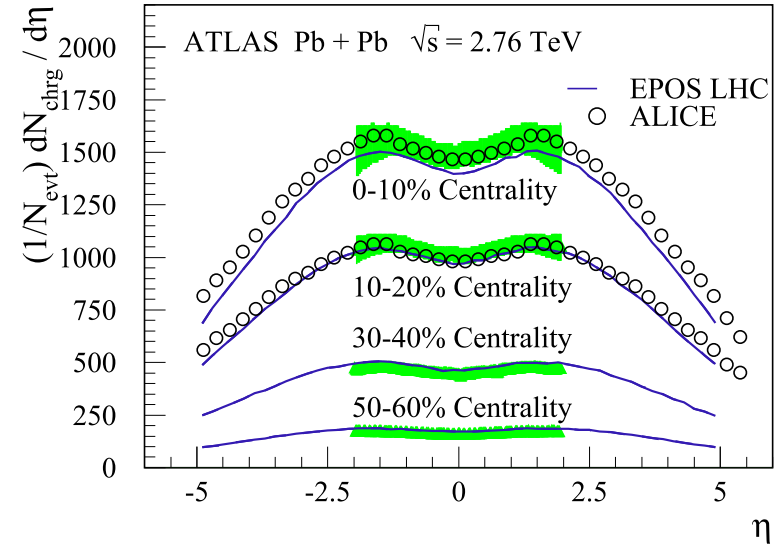

FIG. 17. (Color online) Pseudorapidity distribution of charged particles in $\mathrm{Pb}-\mathrm{Pb}$ collisions at $2.76 \mathrm{TeV} / A$ for centrality bins $0 \%$ to $10 \%, 10 \%$ to $20 \%, 30 \%$ to $40 \%$, and $50 \%$ to $60 \%$. EPOS LHC simulations (solid line) are compared to the ATLAS (band) and ALICE (point) measurements [37,38].

heavy ion data (gamma or $Z$ boson production). This problem is being solved in the EPOS 3 [16] version (currently under development).

\section{B. Proton-lead}

From $p-p$ and $p$-Pb data, all free parameters of Eqs. (4), (5), and (6) are fixed. The free parameter in Eq. (7) is fixed in order to have the same flow in $p$ - $p$ and $p$ - $A$ for the same multiplicity as shown in Fig. 19. Here $p$-Pb simulations at $5 \mathrm{TeV}$ (dashed line) are compared to data and simulations for $p-p$ at $7 \mathrm{TeV}$ as in Fig. 12. At low multiplicity we observe the same flow behavior in $p-p$ and $p$ - $\mathrm{Pb}$ by construction [pp flow parametrization regime from Eq. (8)], but when $N_{c h}$ is higher than about 100 particles, the $\left\langle p_{\mathrm{t}}\right\rangle$ does not increase any more because we enter a different regime with a larger volume, and we have a transition to the AA flow parametrization [from Eq. (5)]. Since $y_{\text {rad }}^{\mathrm{px}} \gg y_{\text {rad }}^{\mathrm{mx}}$, the latter increases much more slowly with the multiplicity.

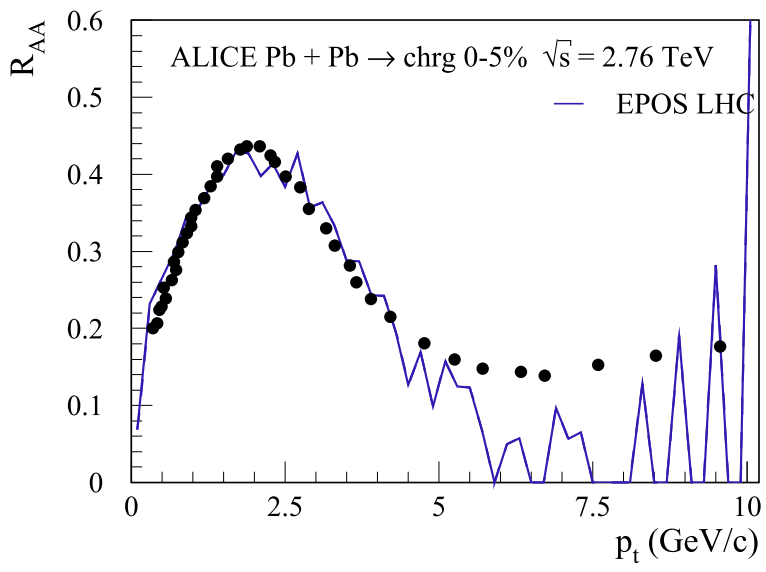

FIG. 18. (Color online) Nuclear modification factor for charged particles in the $5 \%$ most central events of $\mathrm{Pb}-\mathrm{Pb}$ collisions at $2.76 \mathrm{TeV}$. EPOS LHC simulations (solid line) are compared to the ALICE measurements [39] (points).

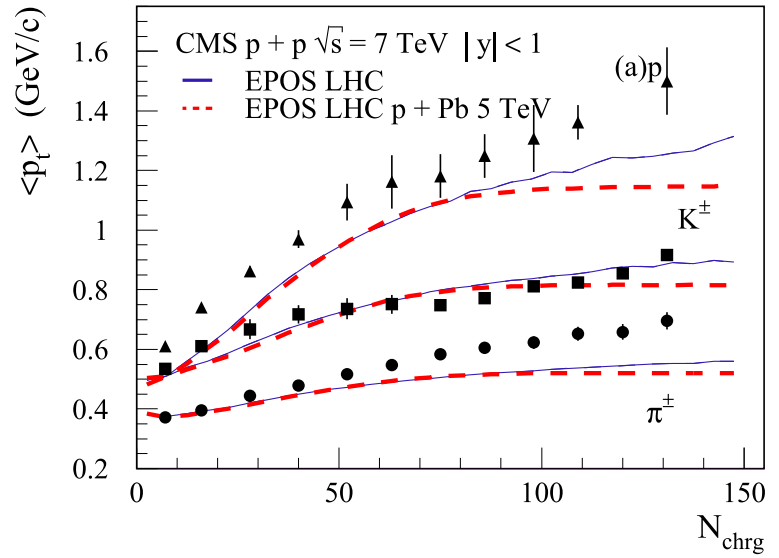

FIG. 19. (Color online) Average transverse momentum $\left\langle p_{t}\right\rangle$ of identified particles $(\pi, K$, and $p$ ) as a function of the number of charged particles for particles with rapidity $|y|<1$ in $p-p$ collisions at $7 \mathrm{TeV}$ (solid line) and $p$ - $\mathrm{Pb}$ collisions at $5 \mathrm{TeV}$ (dashed line). Simulations are done with EPOS LHC including core formation. Points are data from the CMS experiment [29] for $p$ - $p$ scattering.

To test the model predictions, it is now possible to compare to $p-\mathrm{Pb}$ data. As we can see in Fig. 20, the pseudorapidity distribution of charged particles from $p-\mathrm{Pb}$ collisions at $5.02 \mathrm{TeV}$ as measured by the ALICE experiment [40] is very well reproduced by EPOS LHC (solid line). The effect of the core formation on the average multiplicity is very small (dash-dotted line without core formation). It is a real prediction since no parameters have been changed to reproduce these data.

An important test of particle production in nuclear collisions is to study the ratio of the $p_{\mathrm{t}}$ distribution in $p$ - $A$ to the one in $p$ - $p$ normalized by the number of binary collisions. It is called the nuclear modification factor $R_{p \mathrm{~Pb}}$. Any deviation from 1 indicates a nuclear effect. Figure 21 presents the nuclear modification factor of charged particles from $p$-Pb collisions at $5.02 \mathrm{TeV}$ measured by the ALICE Collaboration [41] together

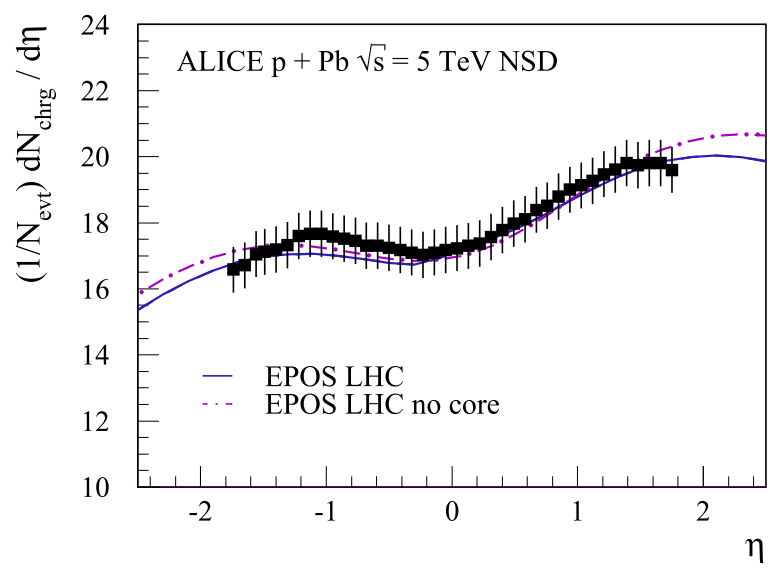

FIG. 20. (Color online) Pseudorapidity distribution of charged particles from $p$ - $\mathrm{Pb}$ collisions at $5.02 \mathrm{TeV}$. Simulations are done with EPOS LHC with (solid line) and without (dash-dotted line) core formation. Points are data from the ALICE experiment [40]. 


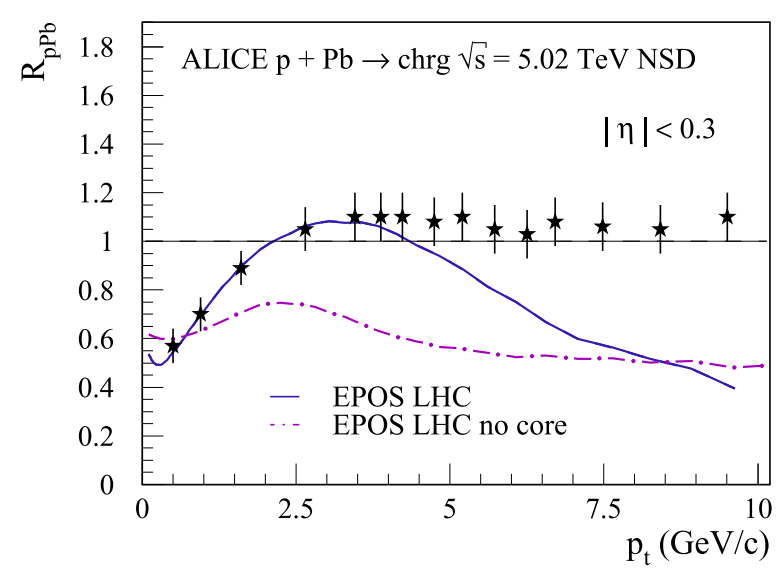

FIG. 21. (Color online) Nuclear modification factor of charged particles from $p-\mathrm{Pb}$ collisions at $5.02 \mathrm{TeV}$. Simulations are done with EPOS LHC, with (solid line) and without (dash-dotted line) core formation. Points are data from the ALICE experiment [41].

with EPOS LHC simulations. Without core formation (dashdotted line) we have a constant $R_{p \mathrm{~Pb}}=0.5$ due to the strong screening in nuclear collisions in EPOS which reduces the number of binary collision in the initial state. This effect is important to get a correct multiplicity but unfortunately the effect is the same for soft and hard processes, leading to a strong suppression of high $p_{\mathrm{t}}$ particles not observed in the data. If the core formation is used (solid line), the situation improves a lot up to $p_{\mathrm{t}} \sim 5 \mathrm{GeV} / c$ but then the strong suppression appears again (as it should since the flow cannot affect high $p_{\mathrm{t}}$ particles).

From the multiplicity measurement it is clear that there is a relative suppression of low $p_{\mathrm{t}}$ particles in $p-\mathrm{Pb}$ relative to $p-p$ and it is now clear that there is no suppression for $p_{\mathrm{t}}>5 \mathrm{GeV} / c$. But the transition region is dominated by the flow effect and the $R_{p \mathrm{~Pb}}=1$ observed for $p_{\mathrm{t}}>2.5 \mathrm{GeV} / c$ has to be interpreted with care because this value is probably unity by chance. If we compare the $R_{p \mathrm{~Pb}}$ for different kinds of particles (light $\pi^{0}$ and heavy proton $p$ ), we can see in Fig. 22

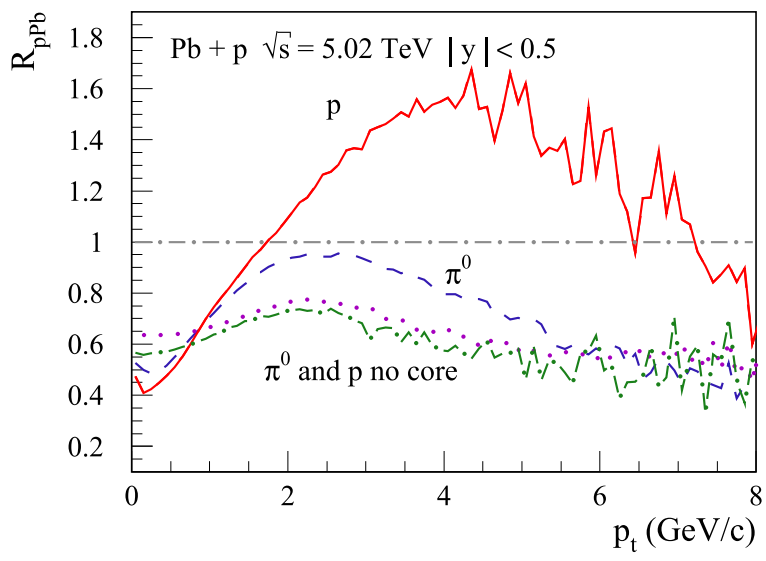

FIG. 22. (Color online) Nuclear modification factor for identified particles $\pi^{0}$ (dashed and dash-dotted lines) and proton $p$ (solid and dashed lines) from $p$-Pb collisions at $5.02 \mathrm{TeV}$. Simulations are done with EPOS LHC with (solid and dashed lines) and without (dotted and dash-dotted lines) core formation. that EPOS predicts that both component will look completely different: mesons having $R_{p P b}<1$ and baryons $R_{p P b}>1$ due to the stronger flow on heavy particles. This can be easily checked with real data, and the effect will be even larger for multistrange baryons.

\section{COMPARISON WITH OTHER MINIMUM BIAS MODELS}

In the following section EPOS LHC is compared to the PYTHIA generator [2], which is commonly used to describe hadron-hadron collisions on an event-by-event basis for cms energies from the Spp $\bar{S}$ to the LHC. The FORTRAN based PYTHIA6 and the newer, $\mathrm{C}++$ based PYTHIA8 version implement very similar soft QCD models; however, the development of PYTHIA6 has stopped and new developments are only implemented in PYTHIA8. Inclusive hadron-hadron collisions are modeled as a superposition of nondiffractive, single-diffractive, and double-diffractive processes, where the different processes are mixed according to their cross sections. The nondiffractive process is calculated at lowestorder perturbative QCD two-to-two parton scatters, where the divergence at $p_{\mathrm{t}} \rightarrow 0$ is regulated via a $p_{\mathrm{t}, \mathrm{min}}$ cutoff. Multiple partonic scatters (MPI) are possible, and their probability is calculated from the geometrical overlap function of the proton matter distribution. Both the matter distribution and the $p_{\mathrm{t}, \mathrm{min}}$ cutoff are tuned to describe the minimum bias and underlying event data at different cms energies. The $p_{\mathrm{t}}$ ordered parton shower algorithm is interleaved with the MPI. A different shower model using virtuality ordering for the emissions and an older MPI model is also available in PYTHIA6, but was never tuned to LHC data. It is therefore not used for comparison.

Fragmentation is implemented via the Lund fragmentation model with free model parameters tuned to Large ElectronPositron (LEP) collider data. The production of heavy quarks is suppressed according to $u: d: s \sim 1: 1: 0.3$, inspired by the quark masses. The rate of $s \bar{s}$ production is a tunable parameter which is usually derived from LEP data. Charm and heavier quarks are not expected to be produced [2].

The hadronization modeling includes a model of color reconnection [42] which is implemented as re-arrangement of strings to minimize their length. Tuning of the free parameters in the color reconnection model yields a very good description of the relation between mean $p_{\mathrm{t}}$ and the charged particle multiplicity as measured at the LHC at a center-of-mass energies of $900 \mathrm{GeV}$ and $7 \mathrm{TeV}$.

Soft diffraction is implemented within a Regge-based Pomeron model to generate the cross section and the diffractive mass and momentum transfer [43,44] with some empirical corrections to cover the full phase space [2]. The soft diffraction model is extended in PYTHIA8 with additional perturbative Pomeron-proton scattering using HERA diffractive parton distribution functions (PDFs) [45].

PYTHIA provides the possibility to alter many modeling details of the phenomenological soft QCD models. A large variety of tunes to minimum bias and underlying event data exist to optimize the descriptions. These tunes vary in the selection of model details, the parton density functions, and the observables they are tuned to; see [47] for a review of tunes 


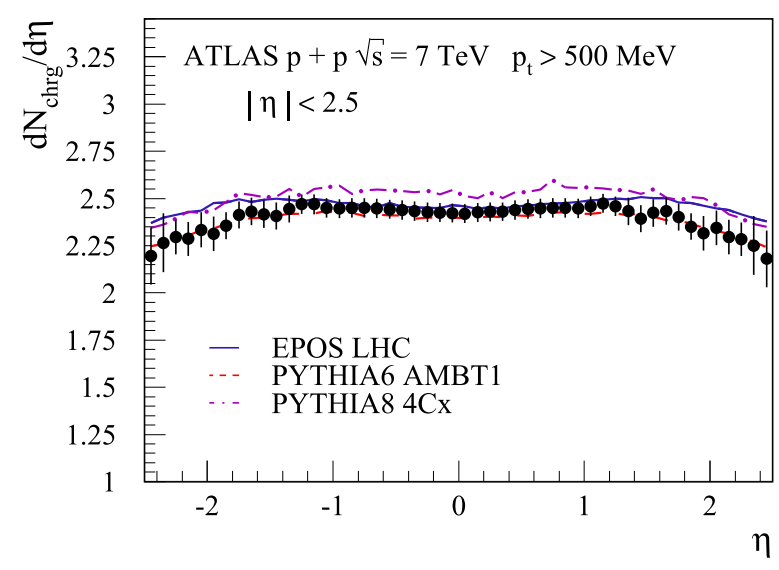

FIG. 23. (Color online) ATLAS measurement of the pseudorapidity distribution of charged particles with $p_{\mathrm{t}} \geqslant 500 \mathrm{MeV}$ in minimum bias collisions [33] compared to PYTHIA6 (dashed line), PYTHIA8 (dash-dotted line), and EPOS LHC (solid line) simulations.

and models. PYTHIA in general yields a very good description of soft QCD effects both in minimum bias data and underlying event measurements at various center-of-mass energies. In the following we compare different LHC measurements to three different setups: EPOS LHC, PYTHIA6 with the AMBT1 tune [33] which was tuned to the ATLAS minimum bias data, and to PYTHIA8 [48] with tune 4Cx [49] which was derived from comparison to minimum bias and underlying event data from LHC. The plots were made using Rivet [50] and are partially taken from MCPLOTS [51].

Figure 23 shows the charged particle production at LHC at $7 \mathrm{TeV}$ with the requirement that the charged particles have a minimum $p_{\mathrm{t}}$ of $500 \mathrm{MeV}$. The data are well described by the PYTHIA6 AMBT1 tune and EPOS LHC, which are both tuned to these data. It is also interesting to note that the PYTHIA6 AMBT1 tune describes the full $\eta$ spectrum while the EPOS LHC tune shows slight deviations towards larger rapidity. The dependence of the charged particle production on the $\mathrm{cms}$ energy of the collision is tunable in PYTHIA, and this energy dependence has been derived from comparing to minimum bias data (and underlying event data for $4 \mathrm{Cx}$ ) at $900 \mathrm{GeV}$ and $7 \mathrm{TeV}$. Good agreement with the data can be reached for all models also at $900 \mathrm{GeV}$ [51]. This also leads to similar predictions for the yet unmeasured charged particle production at the LHC design energy of $14 \mathrm{TeV}$ as shown in Fig. 24.

The slight deviation of PYTHIA8 4Cx in Fig. 23 is caused by the fact that the model is tuned to simultaneously describe the underlying event data shown in Fig. 25. This causes a slightly too high prediction of the particle production in minimum bias events with the current PYTHIA models. A tune to the LHC minimum bias data set alone would give better description as demonstrated in [52]. Figure 25 also shows that EPOS LHC is not able to describe the soft particle production at the LHC when a hard scattering process leading to a jet with $p_{\mathrm{t}}$ above $\sim 5 \mathrm{GeV}$ is involved. This is again due to the fact that that the nonlinear effects as implemented in EPOS LHC affect equally both soft and hard processes. This effect will be corrected in EPOS 3.

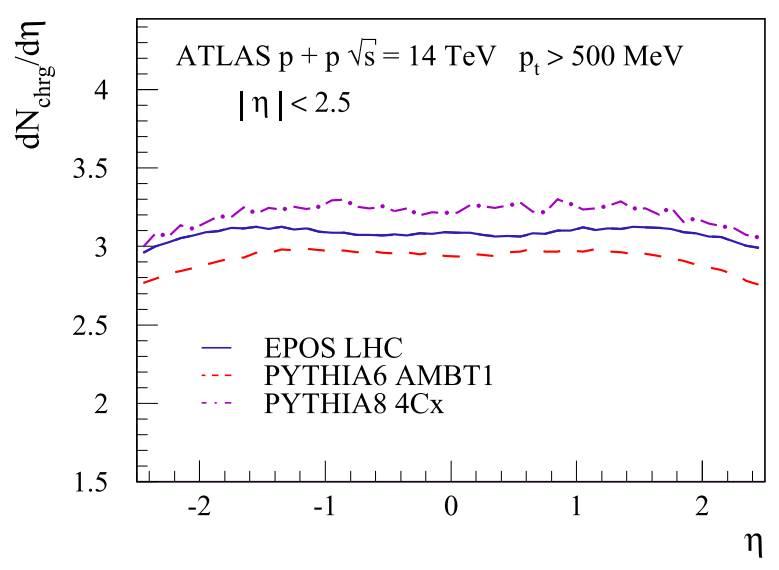

FIG. 24. (Color online) Predicted pseudorapidity distribution of charged particles $p_{\mathrm{t}} \geqslant 500 \mathrm{MeV}$ in minimum bias collisions at the LHC at a cms energy of $14 \mathrm{TeV}$ compared with PYTHIA6 (dashed line), PYTHIA8 (dash-dotted line), and EPOS LHC (solid line) simulations.

Figure 26 shows the same minimum bias data set, but in this case includes charged particles with lower $p_{\mathrm{t}}$, down to $100 \mathrm{MeV}$. EPOS LHC is able to describe both data sets with similar precision, however PYTHIA6 with AMBT1 shows a significantly smaller increase in soft particle production than the data, leading to predictions which are significantly below the data. PYTHIA8 with tune 4Cx describes the data well. It is worth noticing that the difference between PYTHIA6 and EPOS increases if we compare the same distribution at 14 TeV; see Fig. 27. The CMS Collaboration performed a first measurement of the pseudorapidity distribution of charged hadrons with $|\eta|<2$ at $13 \mathrm{TeV}$ without magnetic field and showed that EPOS LHC gives a very good description of these data [53]. Preliminary results from ATLAS confirm this observation [54].

The angular distribution of the charged particle production has also been measured, where the angle is measured with respect to the leading particle. This observable is sensitive

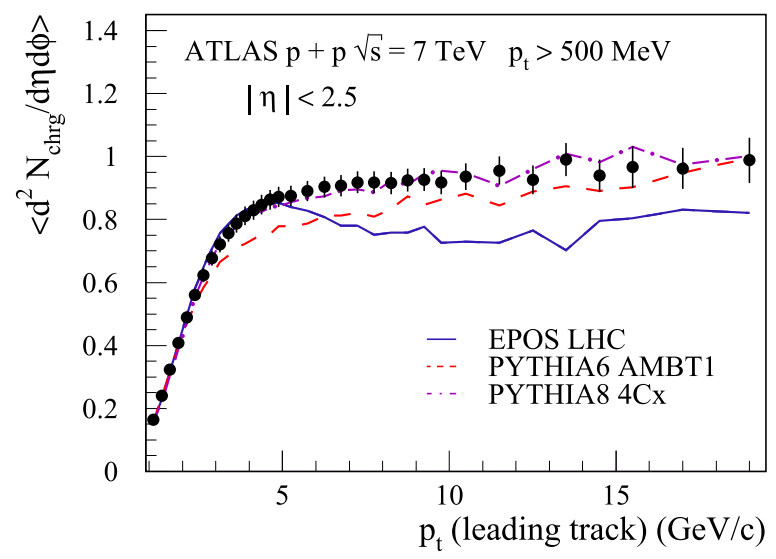

FIG. 25. (Color online) ATLAS measurement of charged particles with $p_{\mathrm{t}} \geqslant 500 \mathrm{MeV}$ produced in the underlying event, i.e., a region of $60^{\circ} \leqslant|\Delta \phi| \leqslant 120^{\circ}$ around the leading particle [46] compared to PYTHIA6 (dashed line), PYTHIA8 (dashed-dotted line), and EPOS LHC (solid line) simulations. 


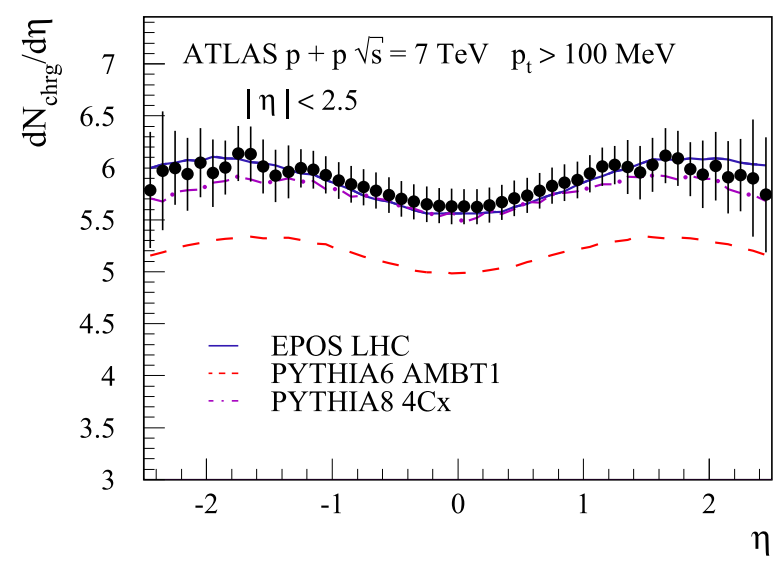

FIG. 26. (Color online) ATLAS measurement of the pseudorapidity distribution of charged particles $p_{\mathrm{t}} \geqslant 100 \mathrm{MeV}$ in minimum bias collisions [33] compared to PYTHIA6 (dashed line), PYTHIA8 (dash-dotted line), and EPOS LHC (solid line) simulations.

to the emergence of jets or so-called minijets in hadronic collisions. The ATLAS Collaboration has measured this distribution with various thresholds for the leading particle $p_{\mathrm{t}}$, ranging from 1.0 to $5 \mathrm{GeV}$. Figure 28 shows the distribution for the lowest $p_{\mathrm{t}}$ threshold, where EPOS LHC is the only generator to provide a very good description both of the total amount and of the shape of the distribution. As the leading particle $p_{\mathrm{t}}$ increases, the description of the data by the PYTHIA models improves, but has up to $20 \%$ deviations close to the leading particle. The description of the data by EPOS LHC gets slightly worse for the higher $p_{\mathrm{t}}$ thresholds, but is always within $10 \%$.

The $p_{\mathrm{t}}$ spectrum of particles in minimum bias events is particularly sensitive to collective flow effects and has been measured by the ATLAS and CMS Collaborations [33,55]. As shown in Fig. 29, both PYTHIA variants have problems describing the $p_{\mathrm{t}}$ spectrum above $\sim 2 \mathrm{GeV}$, despite the effort to tune to these distributions, it seems that the PYTHIA tunes miss an aspect to get a high precision description of these spectra.

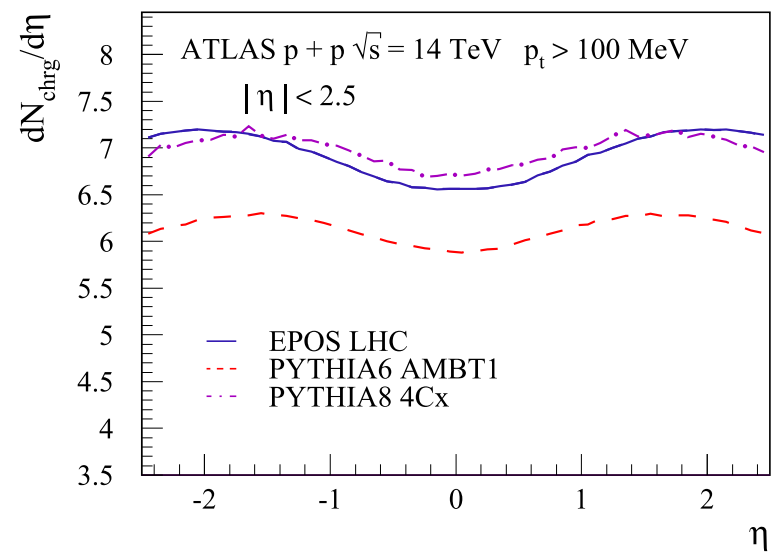

FIG. 27. (Color online) Predicted pseudorapidity distribution of charged particles $p_{\mathrm{t}} \geqslant 100 \mathrm{MeV}$ in minimum bias collisions at the LHC at a cms energy of $14 \mathrm{TeV}$ compared to PYTHIA6 (dashed line), PYTHIA8 (dash-dotted line), and EPOS LHC (solid line) simulations.

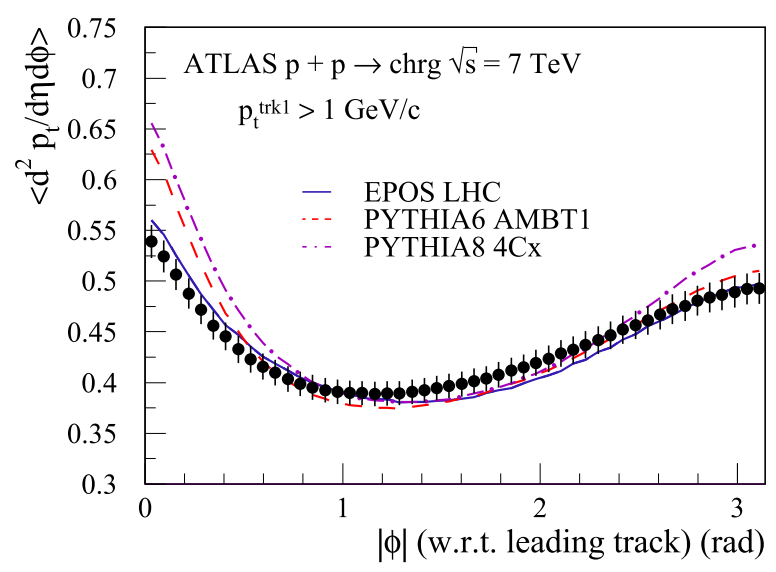

FIG. 28. (Color online) ATLAS measurement of the angular distribution of charged particle production with respect to the leading charged particle with $p_{\mathrm{t}} \geqslant 1 \mathrm{GeV}$ [46] compared to PYTHIA6 (dashed line), PYTHIA8 (dash-dotted line), and EPOS LHC (solid line) simulations.

The EPOS LHC tune describes this spectrum well due to the core with its collective flow; see Fig. 13.

The transverse energy flow, which is sensitive to both the charged and neutral charged particle production, at the LHC has also been measured by the ATLAS Collaboration [56] over the full acceptance range of the detector as shown in Fig. 30. While all models predict a too fast decrease of the transverse energy in the forward region compared to the central region, EPOS LHC describes the data well up to $|\eta|=4.5$. The AMBT1 tune describes the data well in the central region where it was tuned, however only to the charged particles. Apparently the neutral particle production is equally well described by the model. However, in the forward region starting at

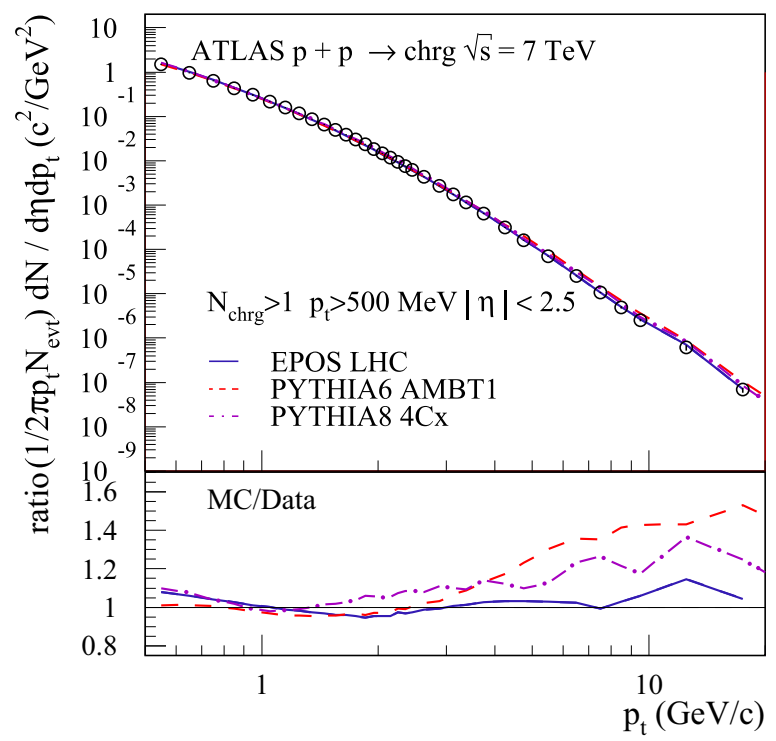

FIG. 29. (Color online) ATLAS measurement of $p_{\mathrm{t}}$ of charged particle produced in minimum bias collisions at $7 \mathrm{TeV}$ [33] compared to PYTHIA6 (dashed line), PYTHIA8 (dash-dotted line), and EPOS LHC (solid line) simulations. 


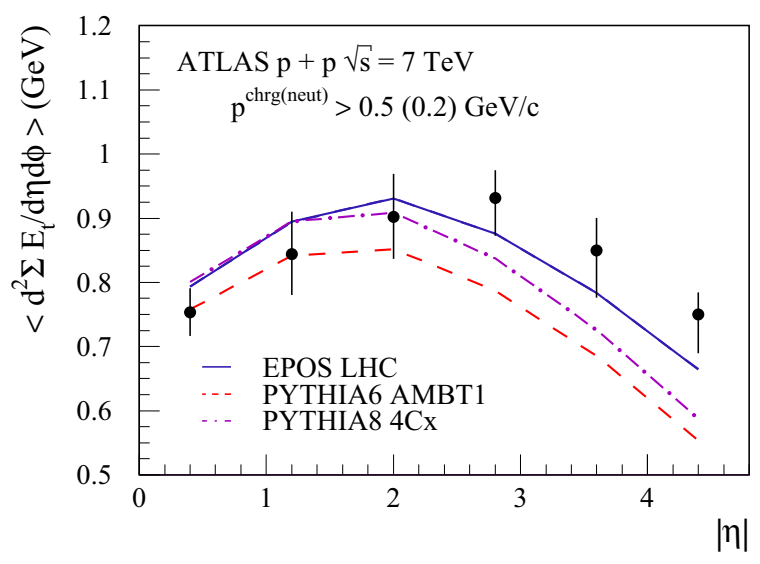

FIG. 30. (Color online) ATLAS measurement of $\sum E_{t}$ as a function of pseudorapidity $\eta$ in minimum bias events at $7 \mathrm{TeV}$ [56] compared to PYTHIA6 (dashed line), PYTHIA8 (dash-dotted line), and EPOS LHC (solid line) simulations.

$|\eta| \geqslant 2.4$, AMBT1 significantly undershoots the data and the disagreement increases towards the forward direction. Tune 4Cx shows a $|\eta|$ dependence similar to AMBT1; however, due to the slightly higher predictions of particle production with $p_{\mathrm{t}} \geqslant 500 \mathrm{MeV}$ as discussed above, it is slightly high in the central region here and is slightly closer to the data in the forward region.

The pseudorapidity gap distribution in minimum bias collisions at $7 \mathrm{TeV}$ has been measured by the ATLAS Collaboration [57]. The cross sections have been measured differentially in terms of $\Delta \eta^{F}$, the larger of the pseudorapidity regions extending to the limits of the ATLAS acceptance, at $\eta= \pm 4.9$, in which no final state particles are produced above a transverse momentum threshold $p_{\mathrm{t}, \text { cut }}$. At small $\Delta \eta^{F}$ the data test the reliability of hadronization models in describing rapidity and transverse momentum fluctuations in final state particle production. The measurements at larger gap sizes are dominated by contributions from the single diffractive dissociation process ( $\mathrm{pp} \rightarrow \mathrm{Xp}$ ), enhanced by double dissociation

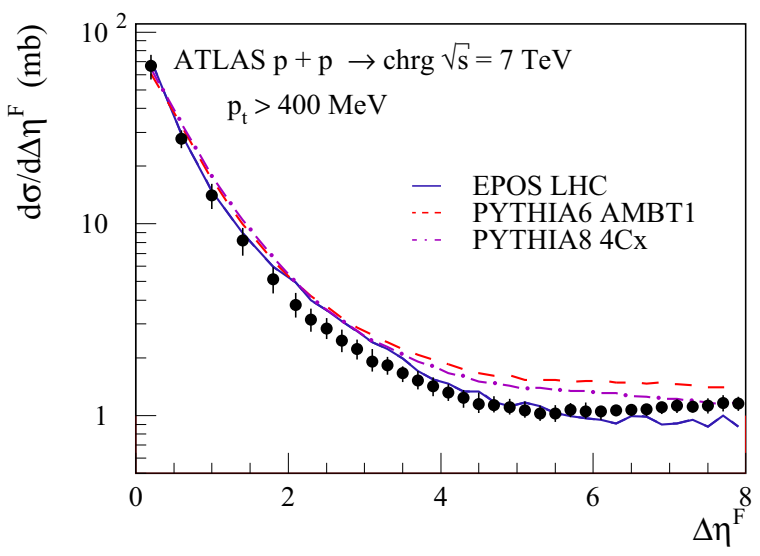

FIG. 31. (Color online) ATLAS measurement of the pseudorapidity gap $\Delta \eta^{F}$ for particles with $p_{\mathrm{t}, \mathrm{cut}}>400 \mathrm{MeV}$ in minimum bias events at $7 \mathrm{TeV}$ [57] compared to EPOS LHC (solid line), PYTHIA6 (dashed line), and PYTHIA8 (dash-dotted line) simulations.

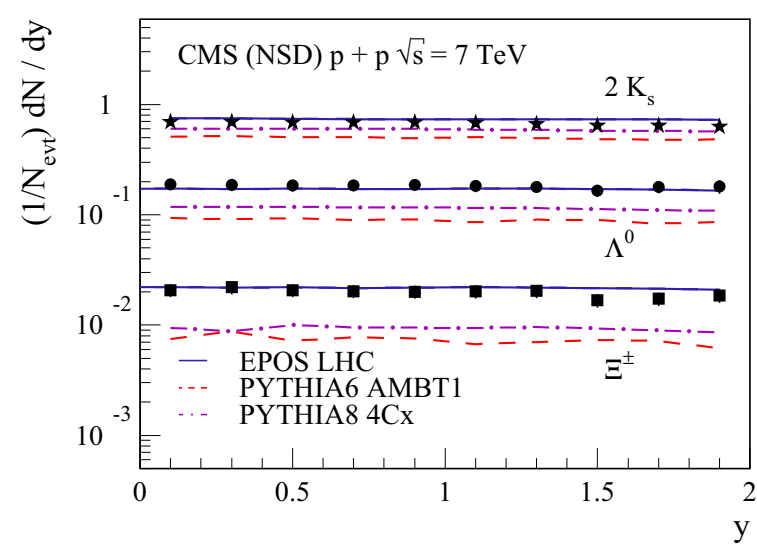

FIG. 32. (Color online) CMS measurement of strange particles $\left(K_{s}^{0}, \Lambda^{0}\right.$, and $\left.\Xi^{ \pm}\right)$yields as a function of rapidity in NSD events at $7 \mathrm{TeV}$ [36] compared to EPOS LHC (solid line), PYTHIA6 (dashed line), and PYTHIA8 (dash-dotted line) simulations.

(pp $\rightarrow \mathrm{XY}$ ). Figure 31 shows the rapidity gap distribution with a $p_{\mathrm{t}, \text { cut }} \geqslant 400 \mathrm{MeV}$. All models describe the small gap region very well, but both PYTHIA tunes overpredict the large gap region where diffractive processes contribute significantly. EPOS LHC, which is tuned to the diffractive cross sections measured at lower energies (SPS) describes this distribution very well.

The EPOS model extensions are also sensitive to the rate and $p_{\mathrm{t}}$ of identified particles $[29,36,58]$, including also particles with strange quarks. Figure 32 shows the rate predictions for $\Lambda, \Xi$, and $K_{s}$ particles as measured by the CMS Collaboration [36]. Both PYTHIA models describe the LEP data on fragmentation well, but apparently fail to describe these LHC data. The same problem is observed with ALICE data on multistrange baryon production [59]. EPOS LHC is able to describe both production results (LEP and LHC) over the full rapidity range due to its statistical hadronization effects combined with string fragmentation. The description of the $p_{\mathrm{t}}$ spectra in Figure 33 is similar: PYTHIA models show

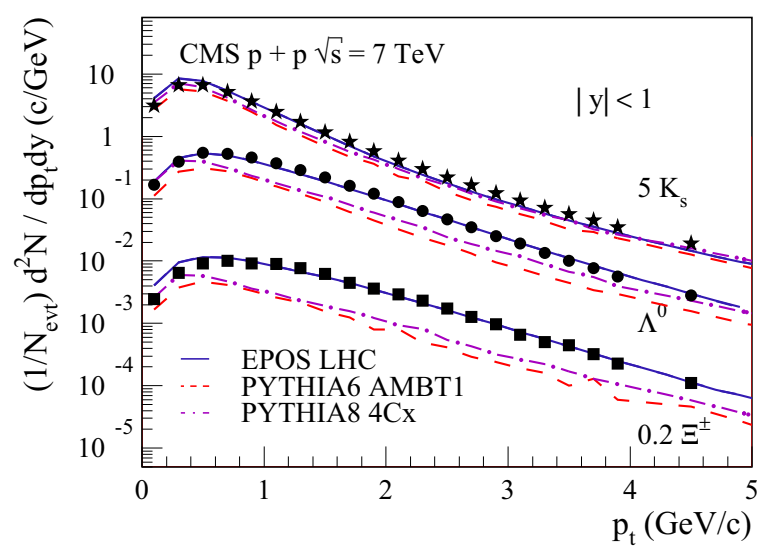

FIG. 33. (Color online) CMS measurement of transverse momentum distribution of strange particles $\left(K_{s}^{0}, \Lambda^{0}\right.$, and $\left.\Xi^{ \pm}\right)$in NSD events at $7 \mathrm{TeV}$ [36] compared to EPOS LHC (solid line), PYTHIA6 (dashed line), and PYTHIA8 (dash-dotted line) simulations. 
significant deviation while EPOS LHC reproduces nicely the data which is attributed to its implementation of collective flow effects.

\section{SUMMARY}

After a short presentation of the main ingredients of the EPOS 1.99 model and in particular the possibility of hadronizing part of the secondary particles including some collective effects, the new flow parametrization implemented in EPOS LHC was described. The main change is that the flow intensity depends only on the total mass of the high density core produced by the overlap of string segments due to multiple parton interactions (MPI in $p$ - $p$ ) or multiple nucleon interactions (MNI dominating in $A-B$ ). Since the volume, and as a consequence the speed of the core expansion, is very different in $p-p$ and $A-B$, this allows two different flow parametrizations to be used for the two different systems. In the case of $p$ - $A$ interactions a smooth transition is used depending on how the core is created (from MPI or MNI). The core decay does not follow usual string fragmentation rules but corresponds to a statistical decay. In addition to the flow, which will have a strong impact on transverse momentum distributions, the particle ratios are modified. In particular, multistrange baryon formation is favored compared to string fragmentation. Comparing EPOS LHC to various LHC data, it is demonstrated that this approach provides a very good description of $p-p, p-\mathrm{Pb}$, and $\mathrm{Pb}-\mathrm{Pb}$ data.

A comparison of the EPOS LHC tunes and different PYTHIA tunes to different LHC measurements was also performed. Similar good agreement can be reached for the pseudorapidity distribution of charged particles and the correlation between mean $p_{\mathrm{t}}$ and multiplicity in the minimum bias events. Significant differences are observed in the $p_{\mathrm{t}}$ spectrum where the EPOS model describes the data well due to its correlated flow treatment, whereas PYTHIA, which lacks such a model, shows up to $20 \%$ deviations. The rate and $p_{\mathrm{t}}$ spectra of the identified particles are well described by EPOS, while significant deviations are observed in PYTHIA. The amount of strange particles is related to the statistical decay in EPOS which can easily create strange quarks at this rate without changing string hadronization constrained by LEP data.

The core formation, including a transverse flow, is also a key point needed to describe in detail even minimum bias $p$ - $p$ data. These effects cannot be neglected in particular in $p$ - $\mathrm{Pb}$ scattering where final state interactions are even more important than in $p$ - $p$. As a consequence any analysis using particles with $p_{\mathrm{t}}<5 \mathrm{GeV} / c$ should be interpreted with care since even $p$ - $p$ data may include final state interactions.
[1] D. d'Enterria, R. Engel, T. Pierog, S. Ostapchenko, and K. Werner, Astropart. Phys. 35, 98 (2011).

[2] T. Sjöstrand, S. Mrenna, and P. Skands, J. High Energy Phys. 05 (2006) 026.

[3] S. G. Matinyan and W. D. Walker, Phys. Rev. D 59, 034022 (1999).

[4] S. Gieseke, C. Rohr, and A. Siodmok, Eur. Phys. J. C 72, 2225 (2012).

[5] P. Z. Skands, Phys. Rev. D 82, 074018 (2010).

[6] K. Werner, F.-M. Liu, and T. Pierog, Phys. Rev. C 74, 044902 (2006).

[7] H. J. Drescher, M. Hladik, S. Ostapchenko, T. Pierog, and K. Werner, Phys. Rep. 350, 93 (2001).

[8] K. Werner, Phys. Rep. 232, 87 (1993).

[9] N. N. Kalmykov, S. S. Ostapchenko, and A. I. Pavlov, in Proceedings, 9th International Symposium on Very High Energy Cosmic Ray Interactions (ISVHECRI 1996) [Nucl. Phys. Proc. Suppl. 52, 17 (1997)].

[10] S. Chatrchyan et al. (CMS Collaboration), Phys. Lett. B 724, 213 (2013).

[11] S. Chatrchyan et al. (CMS Collaboration), Eur. Phys. J. C 74, 2847 (2014).

[12] B. B. Abelev et al. (ALICE Collaboration), Phys. Lett. B 728, 25 (2014).

[13] B. B. Abelev et al. (ALICE Collaboration), Phys. Rev. C 90, 054901 (2014).

[14] T. Pierog and K. Werner, Nucl. Phys. Proc. Suppl. 196, 102 (2009).

[15] K. Werner, I. Karpenko, T. Pierog, M. Bleicher, and K. Mikhailov, Phys. Rev. C 82, 044904 (2010).

[16] K. Werner, B. Guiot, I. Karpenko, and T. Pierog, Phys. Rev. C 89, 064903 (2014).
[17] Available with HepMC interface CRMC at https://web.ikp.kit.edu/rulrich/crmc.html

[18] K. Werner, Iu. Karpenko, and T. Pierog, Phys. Rev. Lett. 106, 122004 (2011).

[19] K. Werner, I. Karpenko, M. Bleicher, T. Pierog, and S. Porteboeuf-Houssais, Phys. Rev. C 85, 064907 (2012).

[20] K. Werner, M. Bleicher, B. Guiot, I. Karpenko, and T. Pierog, Phys. Rev. Lett. 112, 232301 (2014).

[21] F. Becattini and G. Passaleva, Eur. Phys. J. C 23, 551 (2002).

[22] G. Torrieri, S. Steinke, W. Broniowski, W. Florkowski, J. Letessier, and J. Rafelski, Comput. Phys. Commun. 167, 229 (2005).

[23] A. Andronic, P. Braun-Munzinger, K. Redlich, and J. Stachel, Nucl. Phys. A 789, 334 (2007).

[24] P. Huovinen, P. Kolb, U. W. Heinz, P. Ruuskanen, and S. Voloshin, Phys. Lett. B 503, 58 (2001).

[25] K. Werner, Phys. Rev. Lett. 98, 152301 (2007).

[26] R. Baier, Y. L. Dokshitzer, A. H. Mueller, S. Peigne, and D. Schiff, Nucl. Phys. B 483, 291 (1997).

[27] S. Peigne, in Hadronic Physics: Joint Meeting Heidelberg-LigeParis-Wroclaw (HLPW), March 2008, Spa, Belgium, edited by J. Cugnon, J.-P. Lansberg, and N. Matagne, AIP Conf. Proc. No. 1038 (AIP, New York, 2008), p. 139.

[28] K. Werner, I. Karpenko, T. Pierog, M. Bleicher, and K. Mikhailov, Phys. Rev. C 83, 044915 (2011).

[29] S. Chatrchyan et al. (CMS Collaboration), Eur. Phys. J. C 72, 2164 (2012).

[30] T. Csörgö et al. (TOTEM Collaboration), Prog. Theor. Phys. Suppl. 193, 180 (2012).

[31] C. Caso et al. (Particle Data Group), Eur. Phys. J. C 3, 1 (1998).

[32] K. Aamodt et al. (ALICE), Eur. Phys. J. C 68, 345 (2010). 
[33] G. Aad et al. (ATLAS Collaboration), New J. Phys. 13, 053033 (2011).

[34] F. Becattini, J. Cleymans, A. Keranen, E. Suhonen, and K. Redlich, Phys. Rev. C 64, 024901 (2001).

[35] V. Topor Pop, M. Gyulassy, J. Barrette, C. Gale, and A. Warburton, Phys. Rev. C 86, 044902 (2012).

[36] V. Khachatryan et al. (CMS Collaboration), J. High Energy Phys. 05 (2011) 064.

[37] G. Aad et al. (ATLAS Collaboration), Phys. Lett. B710, 363 (2012).

[38] E. Abbas et al. (ALICE Collaboration), Phys. Lett. B 726, 610 (2013).

[39] K. Aamodt et al. (ALICE Collaboration), Phys. Lett. B 696, 30 (2011).

[40] B. Abelev et al. (ALICE Collaboration), Phys. Rev. Lett. 110, 032301 (2013).

[41] B. Abelev et al. (ALICE), Phys. Rev. Lett. 110, 082302 (2013).

[42] P. Z. Skands and D. Wicke, Eur. Phys. J. C 52, 133 (2007).

[43] G. A. Schuler and T. Sjöstrand, Nucl. Phys. B 407, 539 (1993).

[44] G. A. Schuler and T. Sjöstrand, Phys. Rev. D 49, 2257 (1994).

[45] S. Navin, arXiv:1005.3894, Report No. LUTP-09-23,MCNET10-09.

[46] G. Aad et al. (Atlas Collaboration), Phys. Rev. D 83, 112001 (2011).

[47] J. M. Katzy, Prog. Part. Nucl. Phys. 73, 141 (2013).
[48] T. Sjöstrand, S. Mrenna, and P. Z. Skands, Comput. Phys. Commun. 178, 852 (2008).

[49] R. Corke and T. Sjöstrand, J. High Energy Phys. 05 (2011) 009.

[50] A. Buckley, J. Butterworth, L. Lonnblad, D. Grellscheid, H. Hoeth, J. Monk, H. Schulz, and F. Siegert, Comput. Phys. Commun. 184, 2803 (2013).

[51] A. Karneyeu, L. Mijovic, S. Prestel, and P. Skands, Eur. Phys. J. C 74, 2714 (2014).

[52] G. Aad et al. (ATLAS Collaboration), Reports No. ATLPHYS-PUB-2011-009 and No. ATL-COM-PHYS-2011-744, 2011 (unpublished).

[53] V. Khachatryan et al. (CMS Collaboration), arXiv:1507.05915, Reports No. CMS-FSQ-15-001 and No. CERN-PH-EP-2015180.

[54] https://atlas.web.cern.ch/Atlas/GROUPS/PHYSICS/ CONFNOTES/ATLAS-CONF-2015-028/[atlas.web.cern.ch], https://atlas.web.cern.ch/Atlas/GROUPS/PHYSICS/ PUBNOTES/ATL-PHYS-PUB-2015-019/[atlas.web.cern.ch].

[55] V. Khachatryan et al. (CMS Collaboration), Phys. Rev. Lett. 105, 022002 (2010).

[56] G. Aad et al. (ATLAS Collaboration), J. High Energy Phys. 11 (2012) 033.

[57] G. Aad et al. (ATLAS Collaboration), Eur. Phys. J. C 72, 1926 (2012).

[58] G. Aad et al. (ATLAS Collaboration), Phys. Rev. D 85, 012001 (2012).

[59] B. Abelev et al. (ALICE Collaboration), Phys. Lett. B 712, 309 (2012). 